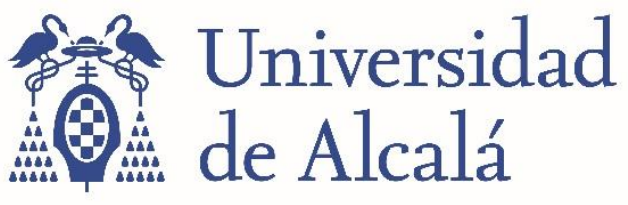

BIBLIOTECA

Document downloaded from the institutional repository of the University of Alcala: http://ebuah.uah.es/dspace/

This is a postprint version of the following published document:

Blasco, A. \& Pérez-Díaz, S. 2020, "A new approach for computing the asymptotes of a parametric curve", Journal of Computational and Applied Mathematics, vol. 364, article 112350

Available at https://doi.org/10.1016/i.cam.2019.112350

(C) 2020 Elsevier

(Article begins on next page)

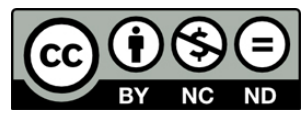

This work is licensed under a

Creative Commons Attribution-NonCommercial-NoDerivatives

4.0 International License. 


\title{
A New Approach for Computing the Asymptotes of a Parametric Curve
}

\author{
Angel Blasco and Sonia Pérez-Díaz \\ Dpto. de Física y Matemáticas \\ Universidad de Alcalá \\ E-28871 Madrid, Spain \\ angel.blasco@uah.es, sonia.perez@uah.es
}

\begin{abstract}
In this paper, we summarize two algorithms for computing all the generalized asymptotes of a plane algebraic curve implicitly or parametrically defined. The approach is based on the notion of perfect curves introduced from the concepts and results presented in previous papers of the same authors. From these results, we derive a new and efficient method that allows to easily compute all the generalized asymptotes of an algebraic curve parametrically defined in $n$-dimensional space.
\end{abstract}

Keywords: Implicit Algebraic Plane Curve; Parametric Plane Curve; Infinity Branches; Asymptotes; Perfect Curves; Approaching Curves.

\section{Introduction}

In this paper we deal with the problem of efficiently computing the asymptotes of an algebraic curve parametrically defined in $n$-dimensional space. This question is very important in the study of real plane algebraic curves because asymptotes contain much of the information about the behavior of the curves in the large. For instance, determining the asymptotes of a curve is an important step in sketching its graph.

Intuitively speaking, the asymptotes of an infinity branch, $B$, of a real plane algebraic curve, $\mathcal{C}$, reflect the status of $B$ at the points with sufficiently large coordinates. In analytic geometry, an asymptote of a curve is a line such that the distance between the curve and the line approaches zero as they tend to infinity. In some contexts, such as algebraic geometry, an asymptote is defined as a line which is tangent to a curve at infinity. 
If $B$ can be defined by some explicit equation of the form $y=f(x)$ (or $x=g(y)$ ), where $f($ or $g$ ) is a continuous function on an infinite interval, it is easy to decide whether $\mathcal{C}$ has an asymptote at $B$ by analyzing the existence of the limits of certain functions when $x$ tends to $\infty$ (or $y$ tends to $\infty$ ). Moreover, if these limits can be computed, we may obtain the equation of the asymptote of $\mathcal{C}$ at $B$. However, if this branch $B$ is implicitly defined and its equation cannot be converted into an explicit form, both the decision and the computation of the asymptote of $\mathcal{C}$ at $B$ require some other tools.

Determining the asymptotes of an implicit algebraic curve is a topic considered in many text-books on analysis (see for instance [6]). A fast and simple method for obtaining the asymptotes of a curve defined by an irreducible polynomial is presented in [5]. In that paper, the emphasis is placed on second order polynomials. Other interesting results can be found in [9]. There, an algorithm, which allows to compute all the linear asymptotes of a real plane algebraic curve $\mathcal{C}$ implicitly defined, is obtained. The asymptotes are represented via polynomial real root isolation.

An algebraic curve may have more general curves than lines describing the status of a branch at the points with sufficiently large coordinates. Intuitively speaking, we say that a curve $\widetilde{\mathcal{C}}$ is a generalized asymptote (or g-asymptote) of another curve $\mathcal{C}$ if the distance between $\widetilde{\mathcal{C}}$ and $\mathcal{C}$ tends to zero as they tend to infinity, and $\mathcal{C}$ can not be approached by a new curve of lower degree (see [1], [2] and [3]). This motivates our interest in efficiently computing these generalized asymptotes.

We have intended the paper to be self-contained. For this reason, we have included Section 2, where we review the theory of infinity branches and introduce the notions of convergent branches (that is, branches that get closer as they tend to infinity) and approaching curves (see [1]), and Section 3, where we lay down fundamental concepts like perfect curve (a curve of degree $d$ that cannot be approached by any curve of degree less than $d$ ) and g-asymptote (a perfect curve that approaches another curve at an infinity branch). In addition, we present an algorithm that obtains the infinity branches of a given curve implicitly defined, and computes a g-asymptote for each of them.

The parametric case is addressed in Section 4 (in particular, see Subsection 4.1). Here, we develop a new method that allows to easily compute all the generalized asymptotes of a rational curve by only determining some simple limits of rational functions constructed from the given parametrization. The results presented are concerned with algebraic plane curves but, as we remark in the paper, they can trivially be adapted for dealing with algebraic curves in $n$-dimensional space (see Example 5).

The method proposed implies the computation of roots of some univariate polynomials, which sometimes may require the use of algebraic numbers. For this reason, we have included some results and examples that show how to overcome this problem by 
using conjugate points and polynomial remainders (see Subsection 4.2). In Subsection 4.3 , we show the advantage of the new algorithm and we report running times for the algorithm presented and the previous algorithms developed by the authors. For this purpose, we consider ten input rational curves defined parametrically (see Section 6). Finally, a section of conclusions and future work is presented (see Section 5).

\section{Notation and previous results}

In this section, we introduce the notion of infinity branch, convergent branches and approaching curves, and we present some properties which allow us to compare the behavior of two implicit algebraic plane curves at infinity. For more details on these concepts and results, we refer to [2] (see Sections 3 and 4).

We consider an irreducible algebraic affine plane curve $\mathcal{C}$ over $\mathbb{C}$ defined by the irreducible polynomial $f(x, y) \in \mathbb{R}[x, y]$. Let $\mathcal{C}^{*}$ be its corresponding projective curve, defined by the homogeneous polynomial

$$
F(x, y, z)=f_{d}(x, y)+z f_{d-1}(x, y)+z^{2} f_{d-2}(x, y)+\cdots+z^{d} f_{0} \in \mathbb{R}[x, y, z],
$$

where $d:=\operatorname{deg}(\mathcal{C})$. We assume that $(0: 1: 0)$ is not an infinity point of $\mathcal{C}^{*}$ (otherwise, we may consider a linear change of coordinates).

In order to get the infinity branches of $\mathbb{C}$, we consider the curve defined by the polynomial $g(y, z)=F(1: y: z)$ and we compute the series expansion for the solutions of $g(y, z)=0$ around $z=0$. There exist exactly $\operatorname{deg}_{y}(g)$ solutions given by different Puiseux series that can be grouped into conjugacy classes. More precisely, if

$$
\varphi(z)=m+a_{1} z^{N_{1} / N}+a_{2} z^{N_{2} / N}+a_{3} z^{N_{3} / N}+\cdots \in \mathbb{C}\langle\langle z\rangle\rangle, \quad a_{i} \neq 0, \forall i \in \mathbb{N},
$$

where $N \in \mathbb{N}, N_{i} \in \mathbb{N}, i \in \mathbb{N}$, and $0<N_{1}<N_{2}<\cdots$, is a Puiseux series such that $g(\varphi(z), z)=0$, and $\nu(\varphi)=N$ (i.e., $N$ is the ramification index of $\varphi$ ), the series

$$
\varphi_{j}(z)=m+a_{1} c_{j}^{N_{1}} z^{N_{1} / N}+a_{2} c_{j}^{N_{2}} z^{N_{2} / N}+a_{3} c_{j}^{N_{3}} z^{N_{3} / N}+\cdots
$$

where $c_{j}^{N}=1, j \in\{1, \ldots, N\}$, are called the conjugates of $\varphi$. The set of all the conjugates of $\varphi$ is called the conjugacy class of $\varphi$ and it contains $\nu(\varphi)$ different series.

Since $g(\varphi(z), z)=0$ in some neighborhood of $z=0$ where $\varphi(z)$ converges, there exists $M \in \mathbb{R}^{+}$such that $F(1: \varphi(t): t)=g(\varphi(t), t)=0$ for $t \in \mathbb{C}$ and $|t|<M$, which implies that $F\left(t^{-1}: t^{-1} \varphi(t): 1\right)=f\left(t^{-1}, t^{-1} \varphi(t)\right)=0$, for $t \in \mathbb{C}$ and $0<|t|<M$. We set $t^{-1}=z$, and we obtain that $f(z, r(z))=0$ for $z \in \mathbb{C}$ and $|z|>M^{-1}$ where

$$
\begin{aligned}
& r(z)=z \varphi\left(z^{-1}\right)=m z+a_{1} z^{1-N_{1} / N}+a_{2} z^{1-N_{2} / N}+a_{3} z^{1-N_{3} / N}+\cdots, \quad a_{i} \neq 0, \forall i \in \mathbb{N} \\
& N, N_{i} \in \mathbb{N}, i \in \mathbb{N} \text {, and } 0<N_{1}<N_{2}<\cdots .
\end{aligned}
$$


Reasoning similarly with the $N$ different series in the conjugacy class, $\varphi_{1}, \ldots, \varphi_{N}$, we get

$$
r_{i}(z)=z \varphi_{i}\left(z^{-1}\right)=m z+a_{1} c_{i}^{N_{1}} z^{1-N_{1} / N}+a_{2} c_{i}^{N_{2}} z^{1-N_{2} / N}+a_{3} c_{i}^{N_{3}} z^{1-N_{3} / N}+\cdots
$$

where $c_{1}, \ldots, c_{N}$ are the $N$ complex roots of $x^{N}=1$. Under these conditions, we introduce the following definition.

Definition 1. An infinity branch of an affine plane curve $\mathcal{C}$ associated to the infinity point $P=(1: m: 0), m \in \mathbb{C}$, is a set $B=\bigcup_{j=1}^{N} L_{j}$, where $L_{j}=\left\{\left(z, r_{j}(z)\right) \in \mathbb{C}^{2}: z \in\right.$ $\mathbb{C},|z|>M\}, M \in \mathbb{R}^{+}$, and

$$
r_{j}(z)=z \varphi_{j}\left(z^{-1}\right)=m z+a_{1} c_{j}^{N_{1}} z^{1-N_{1} / N}+a_{2} c_{j}^{N_{2}} z^{1-N_{2} / N}+a_{3} c_{j}^{N_{3}} z^{1-N_{3} / N}+\cdots
$$

where $N, N_{i} \in \mathbb{N}, i \in \mathbb{N}, 0<N_{1}<N_{2}<\cdots$, and $c_{j}^{N}=1, j \in\{1, \ldots, N\}$. The subsets $L_{1}, \ldots, L_{N}$ are called the leaves of the infinity branch $B$.

Remark 1. An infinity branch is uniquely determined from one leaf, up to conjugation.

That is, if $B=\bigcup_{i=1}^{N} L_{i}$, where $L_{i}=\left\{\left(z, r_{i}(z)\right) \in \mathbb{C}^{2}: z \in \mathbb{C},|z|>M_{i}\right\}$, and

$$
r_{i}(z)=z \varphi_{i}\left(z^{-1}\right)=m z+a_{1} z^{1-N_{1} / N}+a_{2} z^{1-N_{2} / N}+a_{3} z^{1-N_{3} / N}+\cdots
$$

then $r_{j}=r_{i}, j \in\{1, \ldots, N\}$, up to conjugation; i.e.

$$
r_{j}(z)=z \varphi_{j}\left(z^{-1}\right)=m z+a_{1} c_{j}^{N_{1}} z^{1-N_{1} / N}+a_{2} c_{j}^{N_{2}} z^{1-N_{2} / N}+a_{3} c_{j}^{N_{3}} z^{1-N_{3} / N}+\cdots
$$

where $N, N_{i} \in \mathbb{N}$, and $c_{j}^{N}=1, j \in\{1, \ldots, N\}$.

By abuse of notation, we say that $B=\left\{(z, r(z)) \in \mathbb{C}^{2}: z \in \mathbb{C},|z|>M\right\}$ (where $\left.M:=\max \left\{M_{1}, \ldots, M_{N}\right\}\right)$. Moreover, we say that $N$ is the ramification index of the branch $B$ and we write $\nu(B)=N$. Note that $B$ has $\nu(B)$ leaves.

Remark 2. There exists a one-to-one relation between infinity places and infinity branches. In addition, each infinity branch is associated to a unique infinity point given by the center of the corresponding infinity place. More precisely, as we stated above, there exists $M \in \mathbb{R}^{+}$such that $F(1: \varphi(t): t)=g(\varphi(t), t)=0$ for $|t|<M$, where

$$
\varphi(z)=m+a_{1} z^{N_{1} / N}+a_{2} z^{N_{2} / N}+a_{3} z^{N_{3} / N}+\cdots \in \mathbb{C}\langle\langle z\rangle\rangle .
$$

Thus, for $t=0$ we get the infinity point $P=(1: \varphi(0): 0)=(1: m: 0) \in \mathcal{C}^{*}$.

Reciprocally, given an infinity point $P=(1: m: 0)$, there must be, at least, one Puiseux solution $\varphi$ such that $\varphi(0)=m$; this solution provides an infinity branch associated to $P$. Hence, we conclude that every algebraic plane curve has, at least, one infinity branch. 
Remark 3. The proceeding introduced above allows us to obtain the infinity branches of a curve $\mathcal{C}$, under the assumption that $(0: 1: 0) \notin \mathcal{C}^{*}$. However, a curve may have infinity branches, associated to the infinity point $(0: 1: 0)$, which can not be constructed in this way. We call them Type II infinity branches and they have the form $\left\{(r(z), z) \in \mathbb{C}^{2}: z \in \mathbb{C},|z|>M\right\}$. A Type II infinity branch may be obtained by interchanging the variables $x$ and $y$. See [2] (Definition 3.3) for further details.

In the following, we introduce the notions of convergent branches and approaching curves. Intuitively speaking, two infinity branches converge if they get closer as they tend to infinity. This concept will allow us to analyze whether two curves approach each other.

Definition 2. Two infinity branches, $B$ and $\bar{B}$, are convergent if there exist two leaves $L=\left\{(z, r(z)) \in \mathbb{C}^{2}: z \in \mathbb{C},|z|>M\right\} \subset B$ and $\bar{L}=\left\{(z, \bar{r}(z)) \in \mathbb{C}^{2}: z \in \mathbb{C},|z|>\right.$ $\bar{M}\} \subset \bar{B}$ such that $\lim _{z \rightarrow \infty}(\bar{r}(z)-r(z))=0$. In this case, we say that the leaves $L$ and $\bar{L}$ converge.

The following theorem provides a characterization for the convergence of two infinity branches.

Theorem 1. The following statements hold:

1. Two leaves $L=\left\{(z, r(z)) \in \mathbb{C}^{2}: z \in \mathbb{C},|z|>M\right\}$ and $\bar{L}=\left\{(z, \bar{r}(z)) \in \mathbb{C}^{2}: z \in\right.$ $\mathbb{C},|z|>\bar{M}\}$ are convergent if and only if the terms with non negative exponent in the series $r(z)$ and $\bar{r}(z)$ are the same.

2. Two infinity branches $B$ and $\bar{B}$ are convergent if and only if for each leaf $L \subset B$ there exists a leaf $\bar{L} \subset \bar{B}$ convergent with $L$, and reciprocally.

3. Two convergent infinity branches must be associated to the same infinity point.

This paper is concerned with the study of the asymptotes of a curve. The classical concept of asymptote stands for a line that approaches a given curve when it tends to the infinity. In the following we generalize this idea by claiming that two curves approach each other if they, respectively, have two infinity branches that converge (see Definition 3 and Theorem 2 below).

Definition 3. Let $\mathcal{C}$ be an algebraic plane curve with an infinity branch $B$. We say that a curve $\overline{\mathcal{C}}$ approaches $\mathcal{C}$ at its infinity branch $B$ if there exists one leaf $L=\{(z, r(z)) \in$ $\left.\mathbb{C}^{2}: z \in \mathbb{C},|z|>M\right\} \subset B$ such that $\lim _{z \rightarrow \infty} d((z, r(z)), \overline{\mathcal{C}})=0$.

Theorem 2. Let $\mathcal{C}$ be a plane algebraic curve with an infinity branch $B . A$ plane algebraic curve $\overline{\mathcal{C}}$ approaches $\mathcal{C}$ at $B$ if and only if $\overline{\mathcal{C}}$ has an infinity branch, $\bar{B}$, such that $B$ and $\bar{B}$ are convergent. 
Obviously, "approaching" is a symmetric concept, that is, $\mathcal{C}_{1}$ approaches $\mathcal{C}_{2}$ if and only if $\mathcal{C}_{2}$ approaches $\mathcal{C}_{1}$. When it happens we say that $\mathcal{C}_{1}$ and $\mathcal{C}_{2}$ are approaching curves or that they approach each other. In the next section we use this concept to generalize the classical notion of asymptote of a curve.

\section{Asymptotes of an algebraic curve}

Given an algebraic plane curve $\mathcal{C}$ and an infinity branch $B$, in Section 2, we have described how $\mathcal{C}$ can be approached at $B$ by a second curve $\overline{\mathcal{C}}$. Now, suppose that $\operatorname{deg}(\overline{\mathcal{C}})<\operatorname{deg}(\mathcal{C})$. Then one may say that $\mathcal{C}$ degenerates, since it behaves at infinity as a curve of smaller degree. For instance, a hyperbola is a curve of degree 2 that has two real asymptotes, which implies that the hyperbola degenerates, at infinity, to two lines. Similarly, one can check that every ellipse has two asymptotes, although they are complex lines in this case. However, the asymptotic behavior of a parabola is different, since it cannot be approached at infinity by any line. This motivates the following definition:

Definition 4. An algebraic curve of degree $d$ is a perfect curve if it cannot be approached by any curve of degree less than d.

A curve that is not perfect can be approached by other curves of smaller degree. If these curves are perfect, we call them g-asymptotes. More precisely, we have the following definition.

Definition 5. Let $\mathcal{C}$ be a curve with an infinity branch $B$. A g-asymptote (generalized asymptote) of $\mathcal{C}$ at $B$ is a perfect curve that approaches $\mathcal{C}$ at $B$.

The notion of g-asymptote is similar to the classical concept of asymptote. The difference is that a g-asymptote is not necessarily a line, but a perfect curve. Actually, it is a generalization, since every line is a perfect curve (this fact follows from Definition 4). Throughout the paper we refer sometimes to g-asymptote simply as asymptote.

Remark 4. The degree of an g-asymptote is less than or equal to the degree of the curve it approaches. In fact, a g-asymptote of a curve $\mathcal{C}$ at a branch $B$ has minimal degree among all the curves that approach $\mathcal{C}$ at $B$.

In Subsection 3.1, we show that every infinity branch of a given algebraic plane curve implicitly defined has, at least, one asymptote and we show how to compute it. For this purpose, we rewrite Equation 2.1 defining a branch $B$ (see Definition 1) as

$$
r(z)=m z+a_{1} z^{-n_{1} / n+1}+\cdots+a_{k} z^{-n_{k} / n+1}+a_{k+1} z^{-N_{k+1} / N+1}+\cdots
$$

where $0<N_{1}<\cdots<N_{k} \leq N<N_{k+1}<\cdots$ and $\operatorname{gcd}\left(N, N_{1}, \ldots, N_{k}\right)=b, N=n \cdot b$, $N_{j}=n_{j} \cdot b, j \in\{1, \ldots, k\}$. That is, we have simplified the non negative exponents 
such that $\operatorname{gcd}\left(n, n_{1}, \ldots, n_{k}\right)=1$. Note that $0<n_{1}<n_{2}<\cdots$, and $n_{k} \leq n$, and $N<N_{k+1}$, i.e. the terms $a_{j} z^{-N_{j} / N+1}$ with $j \geq k+1$ are those which have negative exponent. We denote these terms as

$$
A(z):=\sum_{\ell=k+1}^{\infty} a_{\ell} z^{-q_{\ell}}, \quad q_{\ell}=-N_{\ell} / N+1 \in \mathbb{Q}^{+}, \quad \ell \geq k+1 .
$$

Under these conditions, we introduce the definition of degree of a branch $B$ :

Definition 6. Let $B=\left\{(z, r(z)) \in \mathbb{C}^{2}: z \in \mathbb{C},|z|>M\right\} \quad(r(z)$ is defined in (3.1)) be an infinity branch associated to an infinity point $P=(1: m: 0), m \in \mathbb{C}$. We say that $n$ is the degree of $B$, and we denote it by $\operatorname{deg}(B)$.

\subsection{Construction of a g-asymptote}

Taking into account Theorems 1 and 2, we have that any curve $\overline{\mathcal{C}}$ approaching $\mathcal{C}$ at $B$ should have an infinity branch $\bar{B}=\left\{(z, \bar{r}(z)) \in \mathbb{C}^{2}: z \in \mathbb{C},|z|>\bar{M}\right\}$ such that the terms with non negative exponent in $r(z)$ and $\bar{r}(z)$ are the same. In the simplest case, if $A=0$ (i.e. there are no terms with negative exponent; see Equation (3.1)), we obtain

$$
\tilde{r}(z)=m z+a_{1} z^{-n_{1} / n+1}+a_{2} z^{-n_{2} / n+1}+\cdots+a_{k} z^{-n_{k} / n+1},
$$

where $a_{1}, a_{2}, \ldots \in \mathbb{C} \backslash\{0\}, m \in \mathbb{C}, n, n_{1}, n_{2} \ldots \in \mathbb{N}, \operatorname{gcd}\left(n, n_{1}, \ldots, n_{k}\right)=1$, and $0<n_{1}<n_{2}<\cdots$. Note that $\tilde{r}$ has the same terms with non negative exponent as $r$, and $\tilde{r}$ does not have terms with negative exponent.

Let $\widetilde{\mathcal{C}}$ be the plane curve containing the branch $\widetilde{B}=\left\{(z, \tilde{r}(z)) \in \mathbb{C}^{2}: z \in \mathbb{C},|z|>\right.$ $\widetilde{M}\}$ (note that $\widetilde{\mathcal{C}}$ is unique since two different algebraic curves have finitely many common points). Observe that

$$
\widetilde{\mathcal{Q}}(t)=\left(t^{n}, m t^{n}+a_{1} t^{n-n_{1}}+\cdots+a_{k} t^{n-n_{k}}\right) \in \mathbb{C}[t]^{2},
$$

where $n, n_{1}, \ldots, n_{k} \in \mathbb{N}, \operatorname{gcd}\left(n, n_{1}, \ldots, n_{k}\right)=1$, and $0<n_{1}<\cdots<n_{k}$, is a polynomial parametrization of $\widetilde{\mathcal{C}}$, and it is proper (see Lemma 3 in [1]). In Theorem 2 in [1], we prove that $\widetilde{\mathcal{C}}$ is a g-asymptote of $\mathcal{C}$ at $B$.

From these results, we obtain the following algorithm, that computes an asymptote for each infinity branch of a given plane curve. We assume that we have prepared the input curve $\mathcal{C}$, by means of a suitable linear change of coordinates, such that $(0: 1: 0)$ is not an infinity point of $\mathcal{C}$. We recall that throughout the paper we refer sometimes to g-asymptote simply as asymptote. 


\section{Algorithm Asymptotes Construction-Implicit Case.}

Given a plane algebraic curve $\mathcal{C}$ implicitly defined by an irreducible polynomial $f(x, y) \in \mathbb{R}[x, y]$, the algorithm computes one asymptote for each of its infinity branches.

1. Compute the infinity points of $\mathcal{C}$. Let $P_{1}, \ldots, P_{n}$ be these points.

2. For each $P_{i}:=\left(1: m_{i}: 0\right)$ do:

2.1. Compute the infinity branches of $\mathcal{C}$ associated to $P_{i}$. Let $B_{i j}=\left\{\left(z, r_{i j}(z)\right) \in\right.$ $\left.\mathbb{C}^{2}: z \in \mathbb{C},|z|>M_{i j}\right\}, j \in\left\{1, \ldots, s_{i}\right\}$, be these branches, where $r_{i j}$ is written as in Equation (3.1). That is,

$$
\begin{gathered}
r_{i j}(z)=m_{i} z+a_{1, i, j} z^{-n_{1, i, j} / n_{i j}+1}+\cdots+a_{k_{i j}, i, j} z^{-n_{k_{i j}, i, j} / n_{i j}+1}+A_{i j}(z), \\
A_{i j}(z)=\sum_{\ell=k_{i j}+1}^{\infty} a_{\ell, i, j} z^{-q_{\ell, i, j}}, \quad q_{\ell, i, j}=-N_{\ell, i, j} / N_{i j}+1 \in \mathbb{Q}^{+}, \ell \geq k_{i j}+1, \\
a_{1, i, j}, a_{2, i, j}, \ldots \in \mathbb{C} \backslash\{0\}, n_{i j}, n_{1, i, j}, \ldots \in \mathbb{N}, 0<n_{1, i, j}<n_{2, i, j}<\cdots, n_{k_{i j}} \leq n_{i j}, \\
N_{i j}<n_{k_{i j}+1}, \text { and } \operatorname{gcd}\left(n_{i j}, n_{1, i, j}, \ldots, n_{k_{i j}, i, j}\right)=1 .
\end{gathered}
$$

2.2. For each branch $B_{i j}, j \in\left\{1, \ldots, s_{i}\right\}$ do:

2.2.1. Consider $\tilde{r}_{i j}$ as in Equation (3.2). That is,

$$
\tilde{r}_{i j}(z)=m_{i} z+a_{1, i, j} z^{-n_{1, i, j} / n_{i j}+1}+\cdots+a_{k_{i j}, i, j} z^{-n_{k_{i j}, i, j} / n_{i j}+1}
$$

Note that $\tilde{r}_{i j}$ has the same terms with non negative exponent as $r_{i j}$, and $\tilde{r}_{i j}$ does not have terms with negative exponent.

2.2.2. Return the asymptote $\widetilde{\mathcal{C}}_{i j}$ defined by the proper parametrization

$$
\widetilde{\mathcal{Q}}_{i j}(t)=\left(t^{n_{i j}}, \tilde{r}_{i j}\left(t^{n_{i j}}\right)\right) \in \mathbb{C}[t]^{2} .
$$

Remark 5. The algorithm Asymptotes Construction-Implicit Case outputs a gasymptote $\widetilde{\mathcal{C}}$ that is independent of the leaf chosen to define the branch $B=\{(z, r(z)) \in$ $\left.\mathbb{C}^{2}: z \in \mathbb{C},|z|>M\right\}$.

Remark 6. In order to compute the implicit equation of the g-asymptote $\widetilde{\mathcal{C}}$, defined parametrically by

$$
\widetilde{\mathcal{Q}}(t)=\left(t^{n_{1}}, a_{n_{2}} t^{n_{2}}+a_{n_{2}-1} t^{n_{2}-1}+\ldots+a_{0}\right),
$$


one may apply for instance the results in [8] (see Chapter 4). More precisely, since $\widetilde{\mathcal{Q}}$ is proper, it holds that

$$
\widetilde{f}(x, y)=\operatorname{resultant}_{t}\left(x-t^{n_{1}}, y-\left(a_{n_{2}} t^{n_{2}}+a_{n_{2}-1} t^{n_{2}-1}+\ldots+a_{0}\right)\right)
$$

is the polynomial that defines implicitly the asymptote $\widetilde{\mathcal{C}}$.

In the following, we illustrate the above algorithm with an example.

Example 1. Let $\mathcal{C}$ be the curve of degree $d=6$ defined by the irreducible polynomial

$f(x, y)=4966 y x+8010 x^{2}-508 x-1335 y^{2} x+421 y^{3} x-4037 y x^{2}+992 y^{2} x^{2}-96 y^{4} x-$ $70 y^{3} x^{2}+75 x^{3}-30 y x^{3}+6 y^{2} x^{3}+6 y^{5} x+53 y^{4}+114 y^{3}+508 y^{2}-24 y^{5}+2 y^{6} \in \mathbb{R}[x, y]$.

We apply algorithm Asymptotes Construction-Implicit Case to compute the asymptotes of $\mathcal{C}$.

Step 1: We have that $f_{6}(x, y)=2 y^{5}(y+3 x)$. Hence, the infinity points are

$$
P_{1}=(1: 0: 0) \quad \text { and } \quad P_{2}=(1:-3: 0) \text {. }
$$

We start by analyzing the point $P_{1}$ :

Step 2.1: There are three infinity branches associated to $P_{1}, B_{1 j}=\left\{\left(z, r_{1 j}(z)\right) \in\right.$ $\left.\mathbb{C}^{2}: z \in \mathbb{C},|z|>M_{1}\right\}, j=1,2,3$, where

$$
\begin{aligned}
& r_{11}(z)=-z^{2 / 3}-4 z^{1 / 3}+7 / 3-5 z^{-1 / 3}+32 / 9 z^{-2 / 3}+4 / 3 z^{-1}+\cdots, \\
& r_{12}(z)=(5 / 2-5 / 2 I)+(-4-7 / 2 I) z^{-1}+(-35 / 4+29 / 2 I) z^{-2}+\cdots, \\
& r_{13}(z)=(5 / 2+5 / 2 I)+(-4+7 / 2 I) z^{-1}+(-35 / 4-29 / 2 I) z^{-2}+\cdots
\end{aligned}
$$

(we compute $r_{1 j}, j=1,2,3$, using the algcurves package included in the computer algebra system Maple; in particular we use the command puiseux).

Step 2.2.1: We compute $\tilde{r}_{1 j}(z), j=1,2,3$, and we have that

$$
\tilde{r}_{11}(z)=-z^{2 / 3}-4 z^{1 / 3}+7 / 3, \tilde{r}_{12}(z)=5 / 2-5 / 2 I, \text { and } \tilde{r}_{13}(z)=5 / 2+5 / 2 I \text {. }
$$

Step 2.2.2: The parametrizations of the asymptotes $\widetilde{\mathcal{C}}_{j}, j=1,2,3$, are given by $\widetilde{\mathcal{Q}}_{1}(t)=\left(t^{3},-t^{2}-4 t+7 / 3\right), \quad \widetilde{\mathcal{Q}}_{2}(t)=(t, 5 / 2-5 / 2 I), \quad \widetilde{\mathcal{Q}}_{3}(t)=(t, 5 / 2+5 / 2 I)$.

One may compute the polynomial defining implicitly $\widetilde{\mathcal{C}_{1}}$ and we have that $\tilde{f}_{1}(x, y)=-27-27 y-54 x-x^{3}-9 x^{2}-3 y x^{2}-18 y x-3 y^{2} x-9 y^{2}-y^{3} \in \mathbb{R}[x, y]$. (apply Remark 6).

Now, we focus on the point $P_{2}$ : 
Step 2.1: The only infinity branch associated to $P_{2}$ is $B_{2}=\left\{\left(z, r_{2}(z)\right) \in \mathbb{C}^{2}: z \in\right.$ $\left.\mathbb{C},|z|>M_{2}\right\}$, where $r_{2}(z)=-3 z+4 z^{-1}-33 / 2 z^{-2}+125 / 2 z^{-3}-1009 / 4 z^{-4}+\cdots$.

Step 2.2.1: We obtain that $\tilde{r}_{2}(z)=-3 z$.

Step 2.2.2: The parametrization of the asymptote $\widetilde{\mathcal{C}}_{4}$ is given by $\widetilde{\mathcal{Q}}_{4}(t)=(t,-3 t) \in$ $\mathbb{R}[t]^{2}$. One may compute the polynomial defining implicitly $\widetilde{\mathcal{C}}_{4}$ (see Remark 6). We have, $\tilde{f}_{4}(x, y)=y+3 x \in \mathbb{R}[x, y]$.

In Figure 1, we plot the curve $\mathcal{C}$, and the asymptotes $\widetilde{\mathcal{C}}_{1}$ and $\widetilde{\mathcal{C}_{4}}$ (the asymptotes $\widetilde{\mathcal{C}}_{2}$ and $\widetilde{\mathcal{C}}_{3}$ are complex lines).
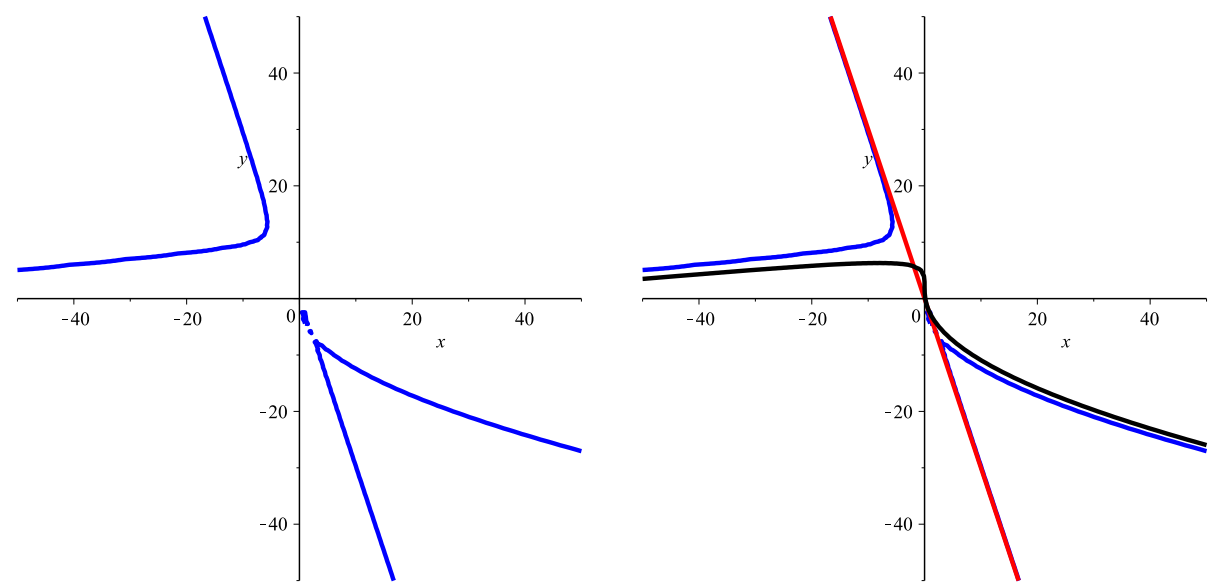

Figure 1: Curve $\mathcal{C}$ (left) and curve and asymptotes (right).

\section{Asymptotes of a parametric curve}

Throughout this paper so far, we have dealt with algebraic plane curves implicitly defined. In this section, we first present a method to compute infinity branches and g-asymptotes of a plane curve from their parametric representation (without implicitizing). This method is included in [3] (see Section 5) and it involves the computation of Puiseux series and infinity branches. In Subsection 4.1, we develop a new method that allows to easily compute the generalized asymptotes (g-asymptotes) by only determining some simple limits of rational functions constructed from the given parametrization. We recall that throughout the paper we refer sometimes to g-asymptote simply as asymptote.

Let $\mathcal{C}$ be a plane curve defined by the parametrization

$$
\mathcal{P}(s)=\left(p_{1}(s), p_{2}(s)\right) \in \mathbb{R}(s)^{2}, \quad p_{i}(s)=p_{i 1}(s) / p_{i 2}(s), \quad \operatorname{gcd}\left(p_{i 1}, p_{i 2}\right)=1, i=1,2 .
$$


If $\mathcal{C}^{*}$ represents the projective curve associated to $\mathcal{C}$, we have that a parametrization of $\mathcal{C}^{*}$ is given by $\mathcal{P}^{*}(s)=\left(p_{1}(s): p_{2}(s): 1\right)$ or, equivalently,

$$
\mathcal{P}^{*}(s)=\left(1: \frac{p_{2}(s)}{p_{1}(s)}: \frac{1}{p_{1}(s)}\right) .
$$

We assume that we have prepared the input curve $\mathcal{C}$, by means of a suitable linear change of coordinates (if necessary) such that $(0: 1: 0)$ is not a point at infinity of $\mathcal{C}^{*}$.

In order to compute the g-asymptotes of $\mathcal{C}$, first we need to determine the infinity branches of $\mathcal{C}$. That is, the sets

$$
B=\left\{(z, r(z)) \in \mathbb{C}^{2}: z \in \mathbb{C},|z|>M\right\}, \text { where } r(z)=z \varphi\left(z^{-1}\right) .
$$

For this purpose, taking into account Definition 1, we have that $f(z, r(z))=F(1$ : $\left.\varphi\left(z^{-1}\right): z^{-1}\right)=F(1: \varphi(t): t)=0$ around $t=0$, where $t=z^{-1}$ and $F$ is the polynomial defining implicitly $\mathcal{C}^{*}$. Observe that in this section, we are given the parametrization $\mathcal{P}^{*}$ of $\mathcal{C}^{*}$ and then, $F\left(\mathcal{P}^{*}(s)\right)=F\left(1: p_{2}(s) / p_{1}(s): 1 / p_{1}(s)\right)=0$. Thus, intuitively speaking, in order to compute the infinity branches of $\mathcal{C}$, and in particular the series $\varphi$, one needs to rewrite the parametrization $\mathcal{P}^{*}(s)$ in the form $(1: \varphi(t): t)$ around $t=0$. For this purpose, the idea is to look for a value of the parameter $s$, say $\ell(t) \in \mathbb{C}\langle\langle t\rangle\rangle$, such that $\mathcal{P}^{*}(\ell(t))=(1: \varphi(t): t)$ around $t=0$.

Hence, from the above reasoning, we deduce that first, we have to consider the equation $1 / p_{1}(s)=t$ (or equivalently, $p_{12}(s)-t p_{11}(s)=0$ ), and we solve it in the variable $s$ around $t=0$. From Puiseux's Theorem, there exist solutions $\ell_{1}(t), \ell_{2}(t), \ldots, \ell_{k}(t) \in \mathbb{C}\langle\langle t\rangle\rangle$ such that, $p_{12}\left(\ell_{i}(t)\right)-t p_{11}\left(\ell_{i}(t)\right)=0, i \in\{1, \ldots, k\}$, in a neighborhood of $t=0$.

Thus, for each $i \in\{1, \ldots, k\}$, there exists $M_{i} \in \mathbb{R}^{+}$such that the points $\left(1: \varphi_{i}(t): t\right)$ or equivalently, the points $\left(t^{-1}: t^{-1} \varphi_{i}(t): 1\right)$, where

$$
\varphi_{i}(t)=\frac{p_{2}\left(\ell_{i}(t)\right)}{p_{1}\left(\ell_{i}(t)\right)},
$$

are in $\mathcal{C}^{*}$ for $|t|<M_{i}$ (note that $\mathcal{P}^{*}(\ell(t)) \in \mathcal{C}^{*}$ since $\mathcal{P}^{*}$ is a parametrization of $\mathcal{C}^{*}$ ). Observe that $\varphi_{i}(t)$ is a Puiseux series, since $p_{2}\left(\ell_{i}(t)\right)$ and $p_{1}\left(\ell_{i}(t)\right)$ can be written as Puiseux series and $\mathbb{C}\langle\langle t\rangle\rangle$ is a field.

Finally, we set $z=t^{-1}$. Then, we have that the points $\left(z, r_{i}(z)\right)$, where $r_{i}(z)=$ $z \varphi_{i}\left(z^{-1}\right)$, are in $\mathcal{C}$ for $|z|>M_{i}^{-1}$. Hence, the infinity branches of $\mathcal{C}$ are the sets $B_{i}=\left\{\left(z, r_{i}(z)\right) \in \mathbb{C}^{3}: z \in \mathbb{C},|z|>M_{i}^{-1}\right\}, \quad i \in\{1, \ldots, k\}$. 
Remark 7. Note that the series $\ell_{i}(t)$ satisfies that $p_{1}\left(\ell_{i}(t)\right) t=1$, for $i \in\{1, \ldots, k\}$. Then, from equality (4.1), we have that

$$
\varphi_{i}(t)=\frac{p_{2}\left(\ell_{i}(t)\right)}{p_{1}\left(\ell_{i}(t)\right)}=p_{2}\left(\ell_{i}(t)\right) t,
$$

and

$$
r_{i}(z)=z \varphi_{i}\left(z^{-1}\right)=p_{2}\left(\ell_{i}\left(z^{-1}\right)\right) .
$$

Once we have the infinity branches, we can compute a g-asymptote for each of them by simply removing the terms with negative exponent from $r_{i}$.

The following algorithm computes the infinity branches of a given parametric space curve and provides an asymptote for each of them.

\section{Algorithm Asymptotes Construction-Parametric Case.}

Given a rational irreducible real algebraic plane curve $\mathcal{C}$ defined by a parametrization $\mathcal{P}(s)=\left(p_{1}(s), p_{2}(s)\right) \in \mathbb{R}(s)^{2}, \quad p_{i}(s)=p_{i 1}(s) / p_{i 2}(s), \quad \operatorname{gcd}\left(p_{i 1}, p_{i 2}\right)=1, i=1,2$, the algorithm outputs one asymptote for each of its infinity branches.

1. Compute the Puiseux solutions of $p_{12}(s)-t p_{11}(s)=0$ around $t=0$. Let them be $\ell_{1}(t), \ell_{2}(t), \ldots, \ell_{k}(t) \in \mathbb{C}\langle\langle t\rangle\rangle$.

2. For each $\ell_{i}(t) \in \mathbb{C}\langle\langle t\rangle\rangle, i \in\{1, \ldots, k\}$, do:

2.1. Compute the corresponding infinity branch of $\mathcal{C}: B_{i}=\left\{\left(z, r_{i}(z)\right) \in \mathbb{C}^{2}\right.$ : $\left.z \in \mathbb{C},|z|>M_{i}\right\}$, where $r_{i}(z)=p_{2}\left(\ell_{i}\left(z^{-1}\right)\right)$ is given as Puiseux series (see Remark 7).

2.2. Consider the series $\tilde{r}_{i}(z)$ obtained by removing the terms with negative exponent in $r_{i}(z)$ (see Equation (3.2)).

2.3. Return the asymptote $\widetilde{\mathcal{C}_{i}}$ defined by the proper parametrization

$$
\widetilde{\mathcal{Q}}_{i}(t)=\left(t^{n_{i}}, \tilde{r}_{i}\left(t^{n_{i}}\right)\right) \in \mathbb{C}[t]^{2} .
$$

where $n_{i}=\operatorname{deg}\left(B_{i}\right)$ (see Definition 6).

Remark 8. 1. In step 1 of the algorithm, some of the solutions $\ell_{1}(t), \ell_{2}(t), \ldots, \ell_{k}(t) \in \mathbb{C}\langle\langle t\rangle\rangle$ might belong to the same conjugacy class. Thus, we only consider one solution for each of these classes. The output asymptote $\widetilde{\mathcal{C}}$ is independent of the solutions $\ell_{1}(t), \ell_{2}(t), \ldots, \ell_{k}(t) \in \mathbb{C}\langle\langle t\rangle\rangle$ chosen in step 1 , and of the leaf chosen to define the branch $B$. 
2. Before to apply the algorithm, the input curve must be prepared such that $(0: 1$ : $0) \notin \mathcal{C}^{*}$ (thus we avoid the possibility that the curve has type II infinity branches; see Definition 3.3 in [2]). As an alternative, one could apply the algorithm first for $\mathcal{P}$ and then for $\overline{\mathcal{P}}:=\left(p_{2}(s), p_{1}(s)\right) \in \mathbb{R}(s)^{2}$. In this last case, if we get the asymptote $\left(h_{1}, h_{2}\right)$, we have to undo the necessary change of coordinates and we finally get the asymptote $\widetilde{\mathcal{Q}}(t)=\left(h_{2}, h_{1}\right)$. Some of the asymptotes obtained from $\overline{\mathcal{P}}$ may coincide with others obtained from $\mathcal{P}$ but some other new asymptotes could appear (those corresponding to vertical asymptotes; see Corollaries 2 and 3) .

In the following example, we consider a parametric plane curve with two infinity branches. We use algorithm Asymptotes Construction-Parametric Case to obtain these branches and compute a g-asymptote for each of them.

Example 2. Let $\mathcal{C}$ be the plane curve defined by the parametrization

$$
\mathcal{P}(s)=\left(\frac{s^{3}+2 s-1}{(s-1)(s-2)^{3}}, \frac{2 s^{3}+s^{2}+1}{(s-2)^{2}(s-1)}\right) \in \mathbb{R}(s)^{2} .
$$

We apply algorithm Asymptotes Construction-Parametric Case to compute the asymptotes of $\mathcal{C}$.

Step 1: We compute the solutions of the equation $p_{12}(s)-t p_{11}(s)=0$ around $t=0$. For this purpose, we may use, for instance, the command puiseux included in the package algcurves of the computer algebra system Maple. There are two solutions that are given by the Puiseux series

$\ell_{1}(t)=1+7102 t^{4}-362 t^{3}+22 t^{2}-2 t, \quad \ell_{2}(t)=2+t+(1 / 11) 11^{2 / 3} t^{2 / 3}+11^{1 / 3} t^{1 / 3}+\cdots$.

\section{Step 2:}

Step 2.1: We compute

$$
\begin{aligned}
& r_{1}(z)=p_{2}\left(\ell_{1}\left(z^{-1}\right)\right)=-2 z-6+50 z^{-1}+\cdots \\
& r_{2}(z)=p_{2}\left(\ell_{2}\left(z^{-1}\right)\right)=\frac{21}{11} \cdot 11^{1 / 3} z^{2 / 3}+\frac{35}{121} \cdot 11^{2 / 3} z^{1 / 3}+\frac{250}{121}-\frac{1852}{1331} \cdot 11^{1 / 3} z^{-1 / 3}+\cdots
\end{aligned}
$$

(we may use, for instance, the command series included in the computer algebra system Maple). The curve has two infinity branches given by $B_{i}=\left\{\left(z, r_{i}(z)\right) \in\right.$ $\left.\mathbb{C}^{2}: z \in \mathbb{C},|z|>M\right\}$ for some $M \in \mathbb{R}^{+}$(note that $B_{2}$ has three leaves).

Step 2.2: We obtain $\tilde{r}_{i}(z)$ by removing the terms with negative exponent in $r_{i}(z)$ for $i=1,2$. We get

$$
\tilde{r}_{1}(z)=-2 z-6 \quad \text { and } \quad \tilde{r}_{2}(z)=21 / 11 \cdot 11^{1 / 3} z^{2 / 3}+35 / 121 \cdot 11^{2 / 3} z^{1 / 3}+250 / 121 .
$$


Step 2.3: The input curve $\mathcal{C}$ has two asymptotes $\widetilde{\mathcal{C}}_{i}$ at $B_{i}$ that can be polynomially parametrized by:

$$
\widetilde{\mathcal{Q}}_{1}(t)=(t,-2 t-6), \quad \widetilde{\mathcal{Q}}_{2}(t)=\left(t^{3}, 21 / 11 \cdot 11^{1 / 3} t^{2}+35 / 121 \cdot 11^{2 / 3} t+250 / 121\right) .
$$

In Figure 2, we plot the curve $\mathcal{C}$, and the asymptotes $\widetilde{\mathcal{C}}_{1}$ and $\widetilde{\mathcal{C}}_{2}$.
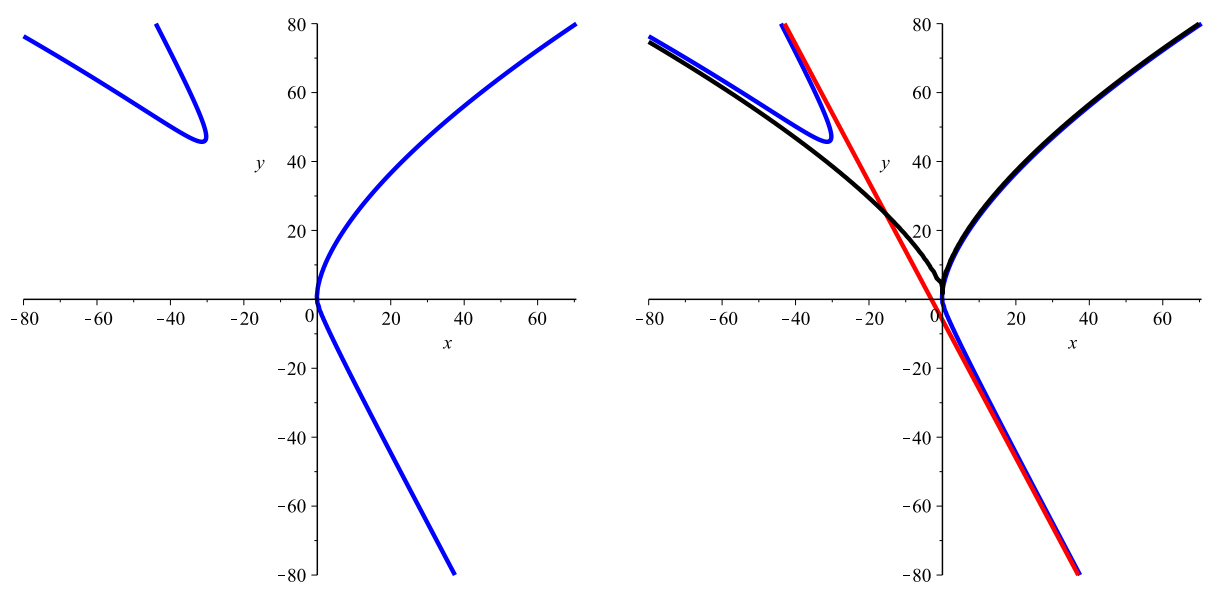

Figure 2: Curve $\mathcal{C}$ (left) and curve and asymptotes (right).

Remark 9. Note that when we compute the series $\ell_{i}$ in step 1 , we cannot handle its infinite terms so it must be truncated, which may distort the computation of the series $r_{i}$ in step 2. However, this distortion may not affect to all the terms in $r_{i}$. In fact, the number of affected terms depends on the number of terms considered in $\ell_{i}$. Nevertheless, note that we do not need to know the full expression of $r_{i}$ but only the terms with non negative exponent. In [3] (see Proposition 2), it is proved that one can get the terms with non negative exponent in $r_{i}$ by considering just $2 \mathrm{deg}\left(p_{1}\right)+1$ terms of $\ell_{i}$.

\subsection{New efficient method for the parametric case}

In this subsection, we present an improvement of the method described above, which avoids the computation of infinity branches and Puiseux series. We develop this method for the plane case but it can be trivially adapted for dealing with rational curves in $n$-dimensional space.

In the following we consider a rational plane curve $\mathcal{C}$ defined by the parametrization

$$
\mathcal{P}(s)=\left(p_{1}(s), p_{2}(s)\right) \in \mathbb{R}(s)^{2}, \quad p_{i}(s)=p_{i 1}(s) / p_{i 2}(s), \quad \operatorname{gcd}\left(p_{i 1}, p_{i 2}\right)=1, i=1,2 .
$$


We assume that $\operatorname{deg}\left(p_{i 1}\right) \leq \operatorname{deg}\left(p_{i 2}\right)=d_{i}, i=1,2$ (otherwise, we apply a linear change of variables); thus, we have that $\lim _{s \rightarrow \infty} p_{i}(s) \neq \infty, i=1,2$ and the infinity branches of $\mathcal{C}$ will be traced when $s$ moves around the different roots of the denominators $p_{12}(s)$ and $p_{22}(s)$. In fact, each of these roots yields an infinity branch. The following theorem shows how to obtain a g-asymptote for each of these branches, by just computing some simple limits of rational functions constructed from $\mathcal{P}(s)$. We recall that throughout the paper we refer sometimes to g-asymptote simply as asymptote.

Theorem 3. Let $\mathcal{C}$ be a curve defined by a parametrization

$$
\mathcal{P}(s)=\left(p_{1}(s), p_{2}(s)\right) \in \mathbb{R}(s)^{2}, \quad p_{i}(s)=p_{i 1}(s) / p_{i 2}(s), \quad \operatorname{gcd}\left(p_{i 1}, p_{i 2}\right)=1, i=1,2,
$$

where $\operatorname{deg}\left(p_{i 1}\right) \leq \operatorname{deg}\left(p_{i 2}\right)=d_{i}, i=1,2$. Let $\tau \in \mathbb{C}$ be such that $p_{i 2}(t)=(t-\tau)^{n_{i}} \bar{p}_{i 2}(t)$ where $\bar{p}_{i 2}(\tau) \neq 0, i=1,2$, and $n_{1} \geq 1$, and let $B$ be the corresponding infinity branch. A g-asymptote of $B$ is defined by the parametrization

$$
\widetilde{\mathcal{Q}}(t)=\left(t^{n_{1}}, a_{n_{2}} t^{n_{2}}+a_{n_{2}-1} t^{n_{2}-1}+\ldots+a_{0}\right)
$$

where

$$
\begin{array}{cc}
a_{n_{2}}=\lim _{t \rightarrow \tau} \frac{p_{2}(t)}{p_{1}(t)^{n_{2} / n_{1}}} & \\
a_{n_{2}-1}=\lim _{t \rightarrow \tau} p_{1}(t)^{1 / n_{1}} f_{1}(t), & f_{1}(t):=\frac{p_{2}(t)}{p_{1}(t)^{n_{2} / n_{1}}}-a_{n_{2}} \\
a_{n_{2}-2}=\lim _{t \rightarrow \tau} p_{1}(t)^{1 / n_{1}} f_{2}(t), & f_{2}(t):=p_{1}(t)^{1 / n_{1}} f_{1}(t)-a_{n_{2}-1} \\
\vdots & \vdots \\
a_{n_{2}-i}=\lim _{t \rightarrow \tau} p_{1}(t)^{1 / n_{1}} f_{i}(t), & f_{i}(t):=p_{1}(t)^{1 / n_{1}} f_{i-1}(t)-a_{n_{2}-(i-1)}, i \in\left\{2, \ldots, n_{2}\right\} .
\end{array}
$$

Proof. First, we recall that at the beginning in Section 4, we have shown how to get the infinite branches of a rational curve by computing the Puiseux solutions of $p_{12}(s)-t p_{11}(s)=0$ around $t=0$. Let $\ell_{1}(t), \ell_{2}(t), \ldots, \ell_{k}(t) \in \mathbb{C}\langle\langle t\rangle\rangle$ be those solutions. For each $i \in\{1, \ldots, k\}$ there exists $M_{i}>0$ such that $p_{12}\left(\ell_{i}(t)\right)-t p_{11}\left(\ell_{i}(t)\right)=0$ for $|t|<M_{i}$; in particular, if $t=0$, we have that $p_{12}\left(\ell_{i}(0)\right)=0$. Thus, each Puiseux solution $\ell_{i}(t)$ is associated to a root of $p_{12}$ given by $\tau_{i}=\ell_{i}(0)$.

Now, we have $\tau \in \mathbb{C}$ such that $p_{i 2}(t)=(t-\tau)^{n_{i}} \bar{p}_{i 2}(t)$ with $\bar{p}_{i 2}(\tau) \neq 0, i=1,2$, and let $\ell(t)$ be the corresponding Puiseux solution. Then, $\ell(0)=\tau$ and we have that

$$
\lim _{s \rightarrow \tau} \mathcal{P}(s)=\lim _{t \rightarrow 0} \mathcal{P}(\ell(t))=\lim _{t \rightarrow 0}\left(p_{1}(\ell(t)), p_{2}(\ell(t))\right)=\lim _{t \rightarrow 0}\left(1 / t, p_{2}(\ell(t))\right)
$$

since, by Remark 7, $p_{1}(\ell(t)) t=1$ in a neighborhood of $t=0$. Furthermore, we can express this limit as follows:

$$
\lim _{t \rightarrow 0}\left(1 / t, p_{2}(\ell(t))\right)=\lim _{z \rightarrow \infty}\left(z, p_{2}(\ell(1 / z))\right)=\lim _{z \rightarrow \infty}(z, r(z)) .
$$


Finally, we observe that a g-asymptote which approaches the infinity branch $B$ is given by

$$
\widetilde{Q}(t)=\left(t^{n}, \widetilde{r}\left(t^{n}\right)\right)=\left(t^{n_{1}}, a_{n_{2}} t^{n_{2}}+a_{n_{2}-1} t^{n_{2}-1}+\ldots+a_{0}\right),
$$

where $\widetilde{r}(z)$ can be computed from $r(z)$ by removing the terms with negative exponent (see Section 3.1). Thus, we have that

$$
\lim _{t \rightarrow \tau} \mathcal{P}(t)=\lim _{z \rightarrow \infty}(z, r(z))=\lim _{z \rightarrow \infty}(z, \widetilde{r}(z))=\lim _{t \rightarrow \infty} \widetilde{Q}(t)
$$

In the following we will use this equality for easily obtaining the coefficients, $a_{n_{2}-i}$ for $i \in\left\{2, \ldots, n_{2}\right\}$, of the polynomial $\widetilde{r}(z)$. Indeed, we have that

$$
\begin{gathered}
\lim _{t \rightarrow \tau} \frac{p_{2}(t)}{p_{1}(t)^{n_{2} / n_{1}}}=\lim _{t \rightarrow \infty} \frac{a_{n_{2}} t^{n_{2}}+a_{n_{2}-1} t^{n_{2}-1}+\ldots+a_{0}}{\left(t^{n_{1}}\right)^{n_{2} / n_{1}}}=a_{n_{2}}, \\
\lim _{t \rightarrow \tau} \frac{p_{2}(t)-a_{n_{2}} p_{1}(t)^{n_{2} / n_{1}}}{p_{1}(t)^{\left(n_{2}-1\right) / n_{1}}}=\lim _{t \rightarrow \infty} \frac{a_{n_{2}-1} t^{n_{2}-1}+\ldots+a_{0}}{\left(t^{n_{1}}\right)^{\left(n_{2}-1\right) / n_{1}}}=a_{n_{2}-1}, \\
\lim _{t \rightarrow \tau} \frac{p_{2}(t)-a_{n_{2}} p_{1}(t)^{n_{2} / n_{1}}-a_{n_{2}-1} p_{1}(t)^{\left(n_{2}-1\right) / n_{1}}}{p_{1}(t)^{\left(n_{2}-2\right) / n_{1}}}=\lim _{t \rightarrow \infty} \frac{a_{n_{2}-2} t^{n_{2}-2}+\ldots+a_{0}}{\left(t^{n_{1}}\right)^{\left(n_{2}-2\right) / n_{1}}}=a_{n_{2}-2}
\end{gathered}
$$

and, reasoning similarly,

$\lim _{t \rightarrow \tau}\left(p_{2}(t)-a_{n_{2}} p_{1}(t)^{n_{2} / n_{1}}-a_{n_{2}-1} p_{1}(t)^{\left(n_{2}-1\right) / n_{1}}-\cdots-a_{1} p_{1}(t)^{1 / n_{1}}\right)=\lim _{t \rightarrow \infty} a_{0}=a_{0}$.

We observe that

$$
\begin{gathered}
\frac{p_{2}(t)-a_{n_{2}} p_{1}(t)^{n_{2} / n_{1}}}{p_{1}(t)^{\left(n_{2}-1\right) / n_{1}}}=p_{1}(t)^{1 / n_{1}} f_{1}(t) \\
\frac{p_{2}(t)-a_{n_{2}} p_{1}(t)^{n_{2} / n_{1}}-a_{n_{2}-1} p_{1}(t)^{\left(n_{2}-1\right) / n_{1}}}{p_{1}(t)^{\left(n_{2}-2\right) / n_{1}}}=p_{1}(t)^{1 / n_{1}} f_{2}(t)
\end{gathered}
$$

and

$$
\left(p_{2}(t)-a_{n_{2}} p_{1}(t)^{n_{2} / n_{1}}-a_{n_{2}-1} p_{1}(t)^{\left(n_{2}-1\right) / n_{1}}-\cdots-a_{1} p_{1}(t)^{1 / n_{1}}\right)=p_{1}(t)^{1 / n_{1}} f_{n_{2}}(t) .
$$

Remark 10. From the above construction, each root $\tau$ of $p_{12}(t)$ yields an infinity branch and, hence, an infinity point $P^{*}$ (see Remark 2). Note that the parametrization $\mathcal{P}(t)$ can be expressed as

$$
\mathcal{P}(t)=\left(\frac{q_{11}(t)}{q(t)}, \frac{q_{12}(t)}{q(t)}\right)
$$

where $q(t)=\operatorname{lcm}\left(p_{12}(t), p_{22}(t)\right)$ and $q_{1 i}(t)=p_{i}(t) q(t)$. Now, the corresponding projective curve is parametrized by $\mathcal{P}^{*}(t)=\left(q_{11}(t), q_{12}(t), q(t)\right)$ and the infinity point associated to $\tau$ is $P^{*}=\left(q_{11}(\tau): q_{12}(\tau): 0\right)$. 
In the following corollaries, we analyze the special case of the vertical and horizontal g-asymptotes, i.e. lines of the form $x-a$ or $y-b$, where $a, b \in \mathbb{C}$ (observe that these asymptotes correspond to branches associated to the infinity points (0:1:0) and $(1: 0: 0)$, respectively). More precisely, we prove that these asymptotes are obtained from the non-common roots of the denominators of the given parametrization. Note that in the practical design of engineering and modeling applications, the rational curves are usually presented by numerical coefficients and $\mathcal{P}(s)$ mostly satisfies that $\operatorname{gcd}\left(p_{12}, p_{22}\right)=1$.

Corollary 1. Let $\mathcal{C}$ be a curve defined by a parametrization

$$
\mathcal{P}(s)=\left(p_{1}(s), p_{2}(s)\right) \in \mathbb{R}(s)^{2}, \quad p_{i}(s)=p_{i 1}(s) / p_{i 2}(s), \quad \operatorname{gcd}\left(p_{i 1}, p_{i 2}\right)=1, i=1,2,
$$

where $\operatorname{deg}\left(p_{i 1}\right) \leq \operatorname{deg}\left(p_{i 2}\right), i=1,2$. Let $\tau \in \mathbb{C}$ be such that $p_{12}(t)=(t-\tau)^{n_{1}} \bar{p}_{12}(t)$ where $p_{22}(\tau) \bar{p}_{12}(\tau) \neq 0$, and $n_{1} \geq 1$. It holds that a g-asymptote of $\mathcal{C}$ corresponding to the infinity point $(1: 0: 0)$ is the horizontal line $y-p_{2}(\tau)=0$, defined by the parametrization $\widetilde{\mathcal{Q}}(t)=\left(t, p_{2}(\tau)\right)$.

Proof. We apply Theorem 3 with $n_{2}=0$.

Corollary 2. Let $\mathcal{C}$ be a curve defined by a parametrization

$$
\mathcal{P}(s)=\left(p_{1}(s), p_{2}(s)\right) \in \mathbb{R}(s)^{2}, \quad p_{i}(s)=p_{i 1}(s) / p_{i 2}(s), \quad \operatorname{gcd}\left(p_{i 1}, p_{i 2}\right)=1, i=1,2,
$$

where $\operatorname{deg}\left(p_{i 1}\right) \leq \operatorname{deg}\left(p_{i 2}\right), i=1,2$. Let $\tau \in \mathbb{C}$ be such that $p_{22}(t)=(t-\tau)^{n_{2}} \bar{p}_{22}(t)$ where $p_{12}(\tau) \bar{p}_{22}(\tau) \neq 0$, and $n_{2} \geq 1$. It holds that a g-asymptote of $\mathcal{C}$ corresponding to the infinity point $(0: 1: 0)$ is the vertical line $x-p_{1}(\tau)=0$, defined by the parametrization $\widetilde{\mathcal{Q}}(t)=\left(p_{1}(\tau), t\right)$.

Proof. We apply Corollary 1 to the parametrization $\left(p_{2}(s), p_{1}(s)\right)$ and we get the asymptote defined by the parametrization $\left(t, p_{1}(\tau)\right)$. Afterwards, we undo the change of coordinates (see statement 2 of Remark 8).

Using the above corollaries, one has the following result that includes Corollaries 1 and 2 .

Corollary 3. Let $\mathcal{C}$ be a curve defined by a parametrization

$$
\mathcal{P}(s)=\left(p_{1}(s), p_{2}(s)\right) \in \mathbb{R}(s)^{2}, \quad p_{i}(s)=p_{i 1}(s) / p_{i 2}(s), \quad \operatorname{gcd}\left(p_{i 1}, p_{i 2}\right)=1, i=1,2,
$$

where $\operatorname{deg}\left(p_{i 1}\right) \leq \operatorname{deg}\left(p_{i 2}\right)=d_{i}, i=1,2$, and such that $p_{i 2}\left(\tau_{i j}\right)=0, i=1,2, j \in$ $\left\{1, \ldots, l_{i}\right\}$ and $p_{i 2}\left(\tau_{k j}\right) \neq 0, i, k \in\{1,2\}, i \neq k, j \in\left\{1, \ldots, l_{i}\right\}$. It holds that the vertical and horizontal asymptotes of $\mathcal{C}$ are the lines

$$
x-p_{1}\left(\tau_{2 j}\right)=0, j \in\left\{1, \ldots, d_{2}\right\}, \quad \text { and } y-p_{2}\left(\tau_{1 j}\right)=0, j \in\left\{1, \ldots, d_{1}\right\} \text {, }
$$

respectively. 
In the following we introduce the Algorithm Improvement Asymptotes ConstructionParametric Case, which uses the above results for computing the g-asymptotes of a rational plane curve.

\section{Algorithm Improvement Asymptotes Construction-Parametric Case.}

Given a rational irreducible real algebraic plane curve $\mathcal{C}$ defined by a parametrization $\mathcal{P}(s)=\left(p_{1}(s), p_{2}(s)\right) \in \mathbb{R}(s)^{2}, \quad p_{i}(s)=p_{i 1}(s) / p_{i 2}(s), \quad \operatorname{gcd}\left(p_{i 1}, p_{i 2}\right)=1, i=1,2$, where $\operatorname{deg}\left(p_{i 1}\right) \leq \operatorname{deg}\left(p_{i 2}\right), i=1,2$, the algorithm outputs one asymptote for each of its infinity branches.

1. Let $\tau_{1}, \ldots, \tau_{k} \in \mathbb{C}$ be the roots of $p_{12}$, and let $n_{11}, \ldots, n_{1 k}$ be their corresponding multiplicities. Let $n_{21}, \ldots, n_{2 k}$ be the multiplicities of these roots in $p_{22}$, respectively.

2. For each $\tau_{i}, i \in\{1, \ldots, k\}$, do:

2.1. Compute

$$
\begin{array}{ccc}
a_{n_{2 i}}=\lim _{t \rightarrow \tau_{i}} \frac{p_{2}(t)}{p_{1}(t)^{n_{2 i} / n_{1 i}}} & \\
a_{n_{2 i}-1}=\lim _{t \rightarrow \tau_{i}} p_{1}(t)^{1 / n_{1 i}} f_{1}(t), & f_{1}(t):=\frac{p_{2}(t)}{p_{1}(t)^{n_{2 i} / n_{1 i}}}-a_{n_{2 i}} \\
a_{n_{2 i}-2}=\lim _{t \rightarrow \tau_{i}} p_{1}(t)^{1 / n_{1 i}} f_{2}(t), & f_{2}(t):=p_{1}(t)^{1 / n_{1 i}} f_{1}(t)-a_{n_{2 i}-1} \\
\vdots & \vdots \\
a_{n_{2 i}-j}=\lim _{t \rightarrow \tau_{i}} p_{1}(t)^{1 / n_{1 i}} f_{j}(t), & f_{j}(t):=p_{1}(t)^{1 / n_{1 i}} f_{j-1}(t)-a_{n_{2 i}-(j-1)},
\end{array}
$$

for $j \in\left\{2, \ldots, n_{2 i}\right\}$.

2.2. Let $\widetilde{\mathcal{C}}_{i}$ be the asymptote defined by the proper parametrization

$$
\widetilde{\mathcal{Q}}_{i}(t)=\left(t^{n_{1 i}}, a_{n_{2 i}} t^{n_{2 i}}+a_{n_{2 i}-1} t^{n_{2 i}-1}+\ldots+a_{0}\right) \in \mathbb{C}[t]^{2} .
$$

3. If there exist $s_{1}, \ldots, s_{l} \in \mathbb{C}$ roots of $p_{22}(s)$ such that $p_{12}\left(s_{j}\right) \neq 0$ for $j \in\{1, \ldots, l\}$ then let $\widetilde{\mathcal{D}}_{i}$ be the vertical asymptote defined by the proper parametrization

$$
\widetilde{\mathcal{M}}_{i}(t)=\left(p_{1}\left(s_{i}\right), t\right) \in \mathbb{C}[t]^{2}, i \in\{1, \ldots, l\}
$$

4. Return the asymptotes $\widetilde{\mathcal{C}}_{1}, \ldots, \widetilde{\mathcal{C}}_{k}$ and $\widetilde{\mathcal{D}}_{1}, \ldots, \widetilde{\mathcal{D}}_{l}$.

Remark 11. In step 2.1 of the algorithm, we compute the coefficients $a_{n_{2 i}-j}$ for $j \in$ $\left\{2, \ldots, n_{2 i}\right\}$. These are the coefficients associated to terms with non negative exponent in the corresponding Puiseux series (see the proof of Theorem 3). Note that we could 
also compute the coefficients associated to terms with negative exponent by recursively applying the same formula for $j>n_{2 i}$. In this way, the whole Puiseux series could be obtained.

By applying Algorithm Improvement Asymptotes Construction-Parametric Case, we can easily obtain all the g-asymptotes of any rational plane curve, as the following examples show.

Example 3. We consider the curve $\mathcal{C}$ introduced in Example 2 and defined by the parametrization

$$
\mathcal{P}(s)=\left(\frac{s^{3}+2 s-1}{(s-1)(s-2)^{3}}, \frac{2 s^{3}+s^{2}+1}{(s-2)^{2}(s-1)}\right) \in \mathbb{R}(s)^{2} .
$$

We apply the algorithm Improvement Asymptotes Construction-Parametric Case.

Step 1: We observe that $p_{12}(s)$ has the roots $\tau_{1}=1, \tau_{2}=2$, with multiplicities $n_{11}=1$ and $n_{12}=3$, respectively. The multiplicities of these roots in $p_{22}(s)$ are $n_{21}=1$ and $n_{22}=2$, respectively.

Step 2: For $\tau_{1}=1$, we compute

$$
\begin{aligned}
& a_{1}=\lim _{t \rightarrow 1} \frac{p_{2}(t)}{p_{1}(t)}=-2 \\
& a_{0}=\lim _{t \rightarrow 1} p_{1}(t) f_{1}(t)=-6, \quad f_{1}(t):=\frac{p_{2}(t)}{p_{1}(t)}-a_{1} .
\end{aligned}
$$

Then, we obtain the asymptote $\widetilde{\mathcal{C}_{1}}$, defined by the proper parametrization

$$
\widetilde{\mathcal{Q}}_{1}(t)=(t,-2 t-6 t) \text {. }
$$

For $\tau_{2}=2$, we compute

$$
\begin{array}{ll}
a_{2}=\lim _{t \rightarrow 2} \frac{p_{2}(t)}{p_{1}(t)^{2}}=\frac{21}{11} \cdot 11^{1 / 3} & \\
a_{1}=\lim _{t \rightarrow 2} p_{1}(t) f_{1}(t)=\frac{35}{121} \cdot 11^{1 / 3}, & f_{1}(t):=\frac{p_{2}(t)}{p_{1}(t)^{2}}-a_{2} \\
a_{0}=\lim _{t \rightarrow 2} p_{1}(t) f_{2}(t)=\frac{250}{121}, & f_{2}(t):=p_{1}(t) f_{1}(t)-a_{1} .
\end{array}
$$

Then, we obtain the asymptote $\widetilde{\mathcal{C}}_{2}$, defined by the proper parametrization

$$
\widetilde{\mathcal{Q}}_{2}(t)=\left(t^{3}, \frac{21}{11} \cdot 11^{1 / 3} t^{2}+\frac{35}{121} \cdot 11^{1 / 3} t+\frac{250}{121}\right) .
$$

Step 3: We observe that all the roots of $p_{12}(t)$ are also roots of $p_{22}(t)$, so there are no vertical asymptotes.

Step 4: The algorithm returns the asymptotes $\widetilde{\mathcal{C}}_{1}$ and $\widetilde{\mathcal{C}}_{2}$.

The input curve, $\mathcal{C}$, and its two g-asymptotes have been plotted in Figure 2. 
Example 4. Let $\mathcal{C}$ be the plane curve defined by the parametrization

$$
\mathcal{P}(s)=\left(\frac{s+1}{s}, \frac{s^{3}-2 s^{2}-3 s+1}{s^{2}(s+1)}\right) \in \mathbb{R}(s)^{2} .
$$

We apply the algorithm Improvement Asymptotes Construction-Parametric Case.

Step 1: We observe that $p_{12}(s)$ has only one root $\tau_{1}=0$, with multiplicity $n_{1}=1$. The multiplicity of this root in $p_{22}$ is $n_{2}=2$.

Step 2: For $\tau_{1}=0$, we compute

$$
\begin{array}{ll}
a_{2}=\lim _{t \rightarrow 0} \frac{p_{2}(t)}{p_{1}(t)^{2}}=1 & \\
a_{1}=\lim _{t \rightarrow 0} p_{1}(t) f_{1}(t)=-6, & f_{1}(t):=\frac{p_{2}(t)}{p_{1}(t)^{2}}-a_{2} \\
a_{0}=\lim _{t \rightarrow 0} p_{1}(t) f_{2}(t)=7, & f_{2}(t):=p_{1}(t) f_{1}(t)-a_{1} .
\end{array}
$$

Then, we obtain the asymptote $\widetilde{\mathcal{C}}$, defined by the proper parametrization $\widetilde{\mathcal{Q}}(t)=\left(t, t^{2}-\right.$ $6 t+7)$. By applying Remark 6 , we have that $\widetilde{\mathcal{C}}$ is defined by the polynomial $\tilde{f}(x, y)=$ $y-x^{2}+6 x-7$.

Step 3: Finally, $s_{1}=-1$ is a root of $p_{22}(s)$ and $p_{12}\left(s_{1}\right) \neq 0$. Thus, additionally to the above asymptotes, we get the vertical asymptote $\widetilde{\mathcal{D}}$ defined by the proper parametrization $\widetilde{\mathcal{M}}(t)=\left(p_{1}(0), t\right)=(0, t)$ or, implicitly, $x=0$.

Step 4: The algorithm returns the asymptotes $\widetilde{\mathcal{C}}$ and $\widetilde{\mathcal{D}}$.
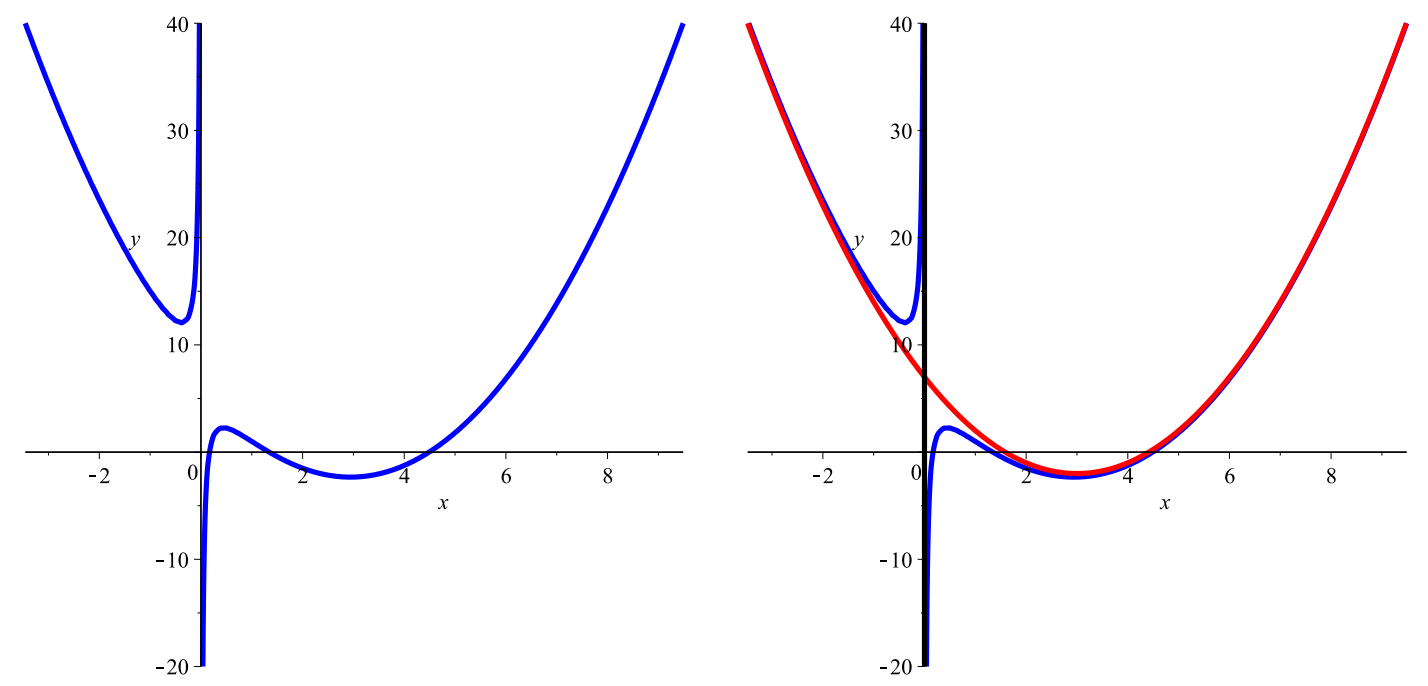

Figure 3: Curve $\mathcal{C}$ (left) and asymptotes and input curve (right). 
The input curve, $\mathcal{C}$, and its two asymptotes have been plotted in Figure 3. Note that the projective parametrization of $\mathcal{C}$ is

$$
\mathcal{P}^{*}(s)=\left(s(s+1)^{2}, s^{3}-2 s^{2}-3 s+1, s^{2}(s+1)\right) \in \mathbb{P}^{2}(\mathbb{R}(s)) .
$$

Since $\mathcal{P}^{*}\left(\tau_{1}\right)=\mathcal{P}^{*}\left(s_{1}\right)=(0: 1: 0)$, from Remark 10, the curve $\mathcal{C}$ has two infinity branches associated to the same infinity point. This means that $P^{*}=(0: 1: 0)$ is a singular infinity point of the curve.

Remark 12. We observe that the method above described may be trivially adapted for dealing with rational algebraic curves in the $n$-dimensional space. For instance, if $n=3$, we have a curve $\mathcal{P}(s)=\left(p_{1}(s), p_{2}(s), p_{3}(s)\right) \in \mathbb{R}(s)^{3}$ with $p_{i}(s)=p_{i 1}(s) / p_{i 2}(s)$ and $\operatorname{gcd}\left(p_{i 1}, p_{i 2}\right)=1, i=1,2,3$, and the asymptotes have the form

$$
\widetilde{\mathcal{Q}}=\left(t^{n_{1}}, a_{n_{2}} t^{n_{2}}+a_{n_{2}-1} t^{n_{2}-1}+\ldots+a_{0}, b_{m_{2}} t^{m_{2}}+b_{m_{2}-1} t^{m_{2}-1}+\ldots+b_{0}\right) .
$$

These asymptotes can be computed by successively applying the algorithm to each component. Note that as in the planar case, roots $\tau \in \mathbb{C}$ such that $p_{22}(\tau)=p_{32}(\tau)=0 \neq p_{12}(\tau)$ could appear (see Step 3 of the algorithm). In this case we must look for asymptotes of the form $\widetilde{\mathcal{M}}=\left(m_{1}(t), t^{n}, m_{3}(t)\right)$ or $\widetilde{\mathcal{M}}=\left(m_{1}(t), m_{2}(t), t^{n}\right)$. Example 5, below, illustrates this ideas.

Example 5. Let $\mathcal{C}$ be the space curve defined by the parametrization

$$
\mathcal{P}(s)=\left(\frac{4\left(s^{2}+1\right)}{(s-2)^{3} s}, \frac{2 s^{2}+2 s-1}{(s-1) s^{2}(s-2)^{2}}, \frac{s+1}{s}\right) \in \mathbb{R}(s)^{3} .
$$

We apply the algorithm Improvement Asymptotes Construction-Parametric Case for obtaining the different g-asymptotes.

Step 1: We observe that $p_{12}(s)$ has the roots $\tau_{1}=0$ and $\tau_{2}=2$, with multiplicities $n_{11}=1$ and $n_{12}=3$, respectively. The multiplicities of these roots in $p_{22}$ are $n_{21}=2$ and $n_{22}=2$, respectively. Finally, the multiplicities of these roots in $p_{32}$ are $n_{31}=1$ and $n_{32}=0$, respectively.

Step 2: For $\tau_{1}=0$, we compute

$$
\begin{array}{ll}
a_{2}=\lim _{t \rightarrow 0} \frac{p_{2}(t)}{p_{1}(t)^{2}}=1 & \\
a_{1}=\lim _{t \rightarrow 0} p_{1}(t) f_{1}(t)=3 / 2, & f_{1}(t):=\frac{p_{2}(t)}{p_{1}(t)^{2}}-a_{2} \\
a_{0}=\lim _{t \rightarrow 0} p_{1}(t) f_{2}(t)=-3 / 2, & f_{2}(t):=p_{1}(t) f_{1}(t)-a_{1},
\end{array}
$$

and

$$
\begin{aligned}
& b_{1}=\lim _{t \rightarrow 0} \frac{p_{3}(t)}{p_{1}(t)}=-2 \\
& b_{0}=\lim _{t \rightarrow 0} p_{1}(t) f_{1}(t)=-1 / 2, \quad f_{1}(t):=\frac{p_{3}(t)}{p_{1}(t)}-b_{1} .
\end{aligned}
$$


Then, we obtain the asymptote $\widetilde{\mathcal{C}_{1}}$, defined by the proper parametrization

$$
\widetilde{\mathcal{Q}}_{1}(t)=\left(t, t^{2}+3 / 2 t-3 / 2,-2 t-1 / 2\right) .
$$

For $\tau_{2}=2$, we compute

$$
\begin{array}{lll}
a_{2}=\lim _{t \rightarrow 2} \frac{p_{2}(t)}{p_{1}(t)^{2 / 3}}=\frac{11}{40} 2^{1 / 3} 5^{1 / 3} & \\
a_{1}=\lim _{t \rightarrow 2} p_{1}(t) f_{1}(t)=-\frac{71}{200} 2^{2 / 3} 5^{2 / 3}, & f_{1}(t):=\frac{p_{2}(t)}{p_{1}(t)^{2}}-a_{2} \\
a_{0}=\lim _{t \rightarrow 2} p_{1}(t) f_{2}(t)=503 / 150, & f_{2}(t):=p_{1}(t) f_{1}(t)-a_{1},
\end{array}
$$

and

$$
b_{0}=\lim _{t \rightarrow 2} p_{3}(t)=p_{3}(2)=3 / 2 .
$$

Then, we obtain the asymptote $\widetilde{\mathcal{C}_{2}}$, defined by the proper parametrization

$$
\widetilde{\mathcal{Q}}_{2}(t)=\left(t, \frac{11}{40} 2^{1 / 3} 5^{1 / 3} t^{2}-\frac{71}{200} 2^{2 / 3} 5^{2 / 3} t+503 / 150,3 / 2\right) .
$$

Step 3: Finally, $s_{1}=1$ is a root of $p_{22}(s)$ and $p_{12}\left(s_{1}\right) p_{32}\left(s_{1}\right) \neq 0$. We deduce that there is an additional asymptote, $\widetilde{\mathcal{D}}$, defined by the proper parametrization

$$
\widetilde{\mathcal{M}}(t)=\left(p_{1}(1), t, p_{3}(1)\right)=(-8, t, 2) .
$$

Step 4: The algorithm returns the asymptotes $\widetilde{\mathcal{C}}_{i}, i=1,2$, and $\widetilde{\mathcal{D}}$.

\subsection{Families of conjugate points}

Algorithm Improvement Asymptotes Construction-Parametric Case allows us to easily obtain all the generalized asymptotes of a rational curve. However, in Step 1 of the algorithm, we compute the roots of the denominators of the parametrization, which may entail certain difficulties if algebraic numbers are involved. In the following, we introduce some ideas, based on the notion of conjugate points (see Definition 12 in [7]), which will help us to overcome this problem.

We start by collecting the points whose coordinates depend algebraically on all the conjugate roots of a same irreducible polynomial, say $m(t) \in \mathbb{R}[t]$. If $m(t)$ is a common factor of both denominators we will need to work with polynomial remainders (see Example 7). Otherwise, Theorem 4, below, tells us how to easily obtain the corresponding asymptotes (from Corollary 3, the curve will have only horizontal or vertical asymptotes in this case). 
Theorem 4. Let $\mathcal{C}$ be a curve defined by a parametrization

$$
\mathcal{P}(s)=\left(p_{1}(s), p_{2}(s)\right) \in \mathbb{R}(s)^{2}, \quad p_{i}(s)=p_{i 1}(s) / p_{i 2}(s), \quad \operatorname{gcd}\left(p_{i 1}, p_{i 2}\right)=1, i=1,2,
$$

where $\operatorname{deg}\left(p_{i 1}\right) \leq \operatorname{deg}\left(p_{i 2}\right)=d_{i}, i=1,2$. The following statements hold:

1. Let $m_{1}(s) \in \mathbb{R}[s]$ be an irreducible polynomial (over $\mathbb{R}$ ) such that $m_{1}(s)$ divides $p_{12}(s)$ and $\operatorname{gcd}\left(m_{1}, p_{22}\right)=1$. Let

$$
R_{1}(y)=\operatorname{resultant}_{s}\left(p_{21}(s)-y p_{22}(s), m_{1}(s)\right) \in \mathbb{R}[s] .
$$

Up to constants in $\mathbb{R}$, it holds that $R_{1}(y)=\prod_{j=1}^{\ell_{1}}\left(y-p_{2}\left(\tau_{1 j}\right)\right)$, where $m_{1}\left(\tau_{1 j}\right)=0$, and $y-p_{2}\left(\tau_{1 j}\right)$ are the horizontal asymptotes for $j \in\left\{1, \ldots, \ell_{1}\right\}$.

2. Let $m_{2}(s) \in \mathbb{R}[s]$ be an irreducible polynomial (over $\mathbb{R}$ ) such that $m_{2}(s)$ divides $p_{22}(s)$ and $\operatorname{gcd}\left(m_{2}, p_{12}\right)=1$. Let

$$
R_{2}(x)=\operatorname{resultant}_{s}\left(p_{11}(s)-x p_{12}(s), m_{2}(s)\right) \in \mathbb{R}[s] .
$$

Up to constants in $\mathbb{R}$, it holds that $R_{2}(x)=\prod_{j=1}^{\ell_{2}}\left(x-p_{1}\left(\tau_{2 j}\right)\right)$, where $m_{2}\left(\tau_{2 j}\right)=0$, and $x-p_{1}\left(\tau_{2 j}\right)$ are the vertical asymptotes for $j \in\left\{1, \ldots, \ell_{2}\right\}$.

Proof. We prove statement 1 (for statement 2, one reasons similarly). By applying the resultant properties (see e.g. Appendix 2 in [8]), one has that

$$
R_{1}(y)=\operatorname{lc}\left(m_{1}\right)^{k} \prod_{j=1}^{\ell_{1}}\left(p_{21}\left(\tau_{1 j}\right)-y p_{22}\left(\tau_{1 j}\right)\right)
$$

where $k=\operatorname{deg}_{s}\left(p_{21}(s)-y p_{22}(s)\right)$, and $\operatorname{lc}\left(m_{1}\right)$ denotes the leading coefficient of the polynomial $m_{1}$. Thus, up to constants in $\mathbb{R}$, we may write $R_{1}(y)=\prod_{j=1}^{\ell_{1}}\left(y-p_{2}\left(\tau_{1 j}\right)\right)$. Note that, from Corollary 1 , we get that $y-p_{2}\left(\tau_{1 j}\right)$ are the horizontal asymptotes for $j \in\left\{1, \ldots, \ell_{1}\right\}$.

The following example shows how we can apply Theorem 4 to obtain the horizontal and vertical asymptotes of a rational plane curve.

Example 6. Let $\mathcal{C}$ be the plane curve defined by the parametrization

$$
\mathcal{P}(s)=\left(\frac{4\left(s^{2}+1\right)}{\left(s^{3}+s+1\right)(s-2)^{3} s}, \frac{2 s^{3}+s+1}{(s-1) s^{2}(s-2)^{2}}\right) \in \mathbb{R}(s)^{2} .
$$

We apply the algorithm Improvement Asymptotes Construction-Parametric Case.

Step 1: We have that $\tau_{1}=0, \tau_{2}=2$ are two real roots of $p_{12}(s)$ of multiplicity $n_{11}=1, n_{12}=3$, respectively. In addition, $n_{21}=2, n_{22}=2$ are the multiplicities 
of these roots in $p_{22}$, respectively. We also have one real and two complex roots of $p_{12}(s)$ corresponding to the roots of the polynomial $m_{1}(s)=s^{3}+s+1$. These roots have multiplicity 1 in $p_{12}(s)$ and multiplicity 0 in $p_{22}(s)$.

We note that $\tau_{1}$ corresponds to an infinity branch which is associated to the infinity point $(0: 1: 0)$. On the other hand, $\tau_{2}$ and the roots of the polynomial $m_{1}(s)$ provide two infinity branches associated to the infinity point (1:0:0) (see Remark 10).

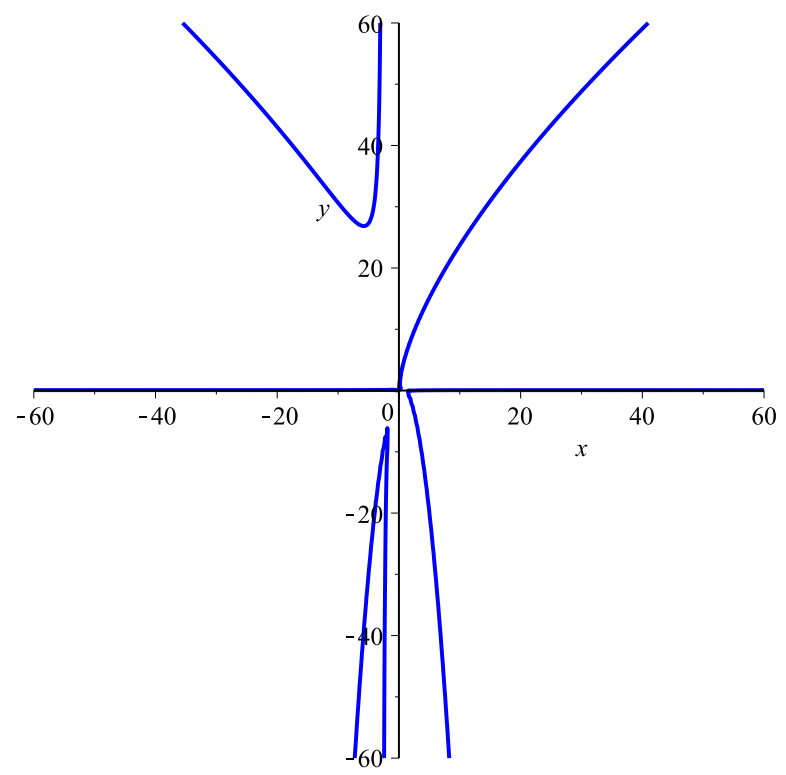

Figure 4: The curve $\mathcal{C}$ has four real and two complex infinity branches.

\section{Step 2:}

- For $\tau_{1}=0$, we compute

$$
\begin{array}{ll}
a_{2}=\lim _{t \rightarrow 0} \frac{p_{2}(t)}{p_{1}(t)^{2}}=-1 & \\
a_{1}=\lim _{t \rightarrow 0} p_{1}(t) f_{1}(t)=1, & f_{1}(t):=\frac{p_{2}(t)}{p_{1}(t)^{2}}-a_{2} \\
a_{0}=\lim _{t \rightarrow 0} p_{1}(t) f_{2}(t)=1 / 8, & f_{2}(t):=p_{1}(t) f_{1}(t)-a_{1} .
\end{array}
$$

Then, we obtain the asymptote $\widetilde{\mathcal{C}}_{1}$, defined by the proper parametrization $\widetilde{\mathcal{Q}}_{1}(t)=$ $\left(t,-t^{2}+t+1 / 8\right)$. By applying Remark 6 , we have that $\widetilde{\mathcal{C}}_{1}$ is defined by the polynomial $\tilde{f}_{1}(x, y)=y+x^{2}-x-1 / 8$ (see Figures 4 and 5 ).

- For $\tau_{2}=2$, we compute

$$
\begin{array}{ll}
a_{2}=\lim _{t \rightarrow 2} \frac{p_{2}(t)}{p_{1}(t)^{2 / 3}}=\frac{19}{40} 2^{1 / 3} 605^{1 / 3} & \\
a_{1}=\lim _{t \rightarrow 2} p_{1}(t)^{1 / 3} f_{1}(t)=\frac{-151}{36300} 2^{2 / 3} 605^{2 / 3}, & f_{1}(t):=\frac{p_{2}(t)}{p_{1}(t)^{2 / 3}}-a_{2} \\
a_{0}=\lim _{t \rightarrow 2} p_{1}(t)^{1 / 3} f_{2}(t)=\frac{152983}{72600}, & f_{2}(t):=p_{1}(t)^{1 / 3} f_{1}(t)-a_{1} .
\end{array}
$$



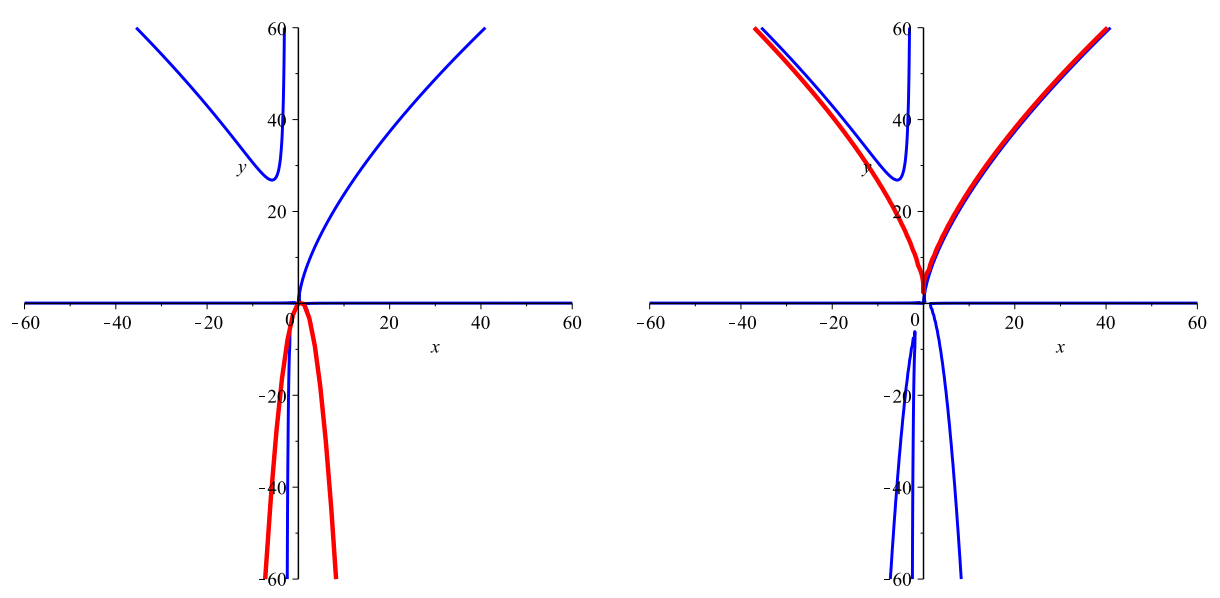

Figure 5: Curve $\mathcal{C}$ and asymptote $\widetilde{\mathcal{C}_{1}}$ (left), curve and asymptote $\widetilde{\mathcal{C}_{2}}$ (right).

Then, we obtain the asymptote $\widetilde{\mathcal{C}}_{2}$, defined by the proper parametrization

$$
\widetilde{\mathcal{Q}}_{2}(t)=\left(t^{3}, \frac{19}{40} 2^{1 / 3} 605^{1 / 3} t^{2}-\frac{151}{36300} 2^{2 / 3} 605^{2 / 3} t+\frac{152983}{72600}\right) .
$$

By applying Remark 6, we have that $\widetilde{\mathcal{C}}_{2}$ is defined by the polynomial

$$
\begin{aligned}
& \tilde{f}_{2}(x, y)=-829939 / 6400 x^{2}+2869 / 400 y x-784526087 / 52272000 x+ \\
& y^{3}-152983 / 24200 y^{2}+23403798289 / 1756920000 y- \\
& 3580383273646087 / 382657176000000
\end{aligned}
$$

(see Figures 4 and 5).

- Finally, we apply Theorem 4 to compute the asymptotes obtained from the polynomial $m_{1}(s)=s^{3}+s+1$. Since $m_{1}(s)$ divides $p_{12}(s)$ and $\operatorname{gcd}\left(m_{1}, p_{22}\right)=1$, we have that

$$
R_{1}(y)=\operatorname{resultant}_{s}\left(p_{21}(s)-y p_{22}(s), m_{1}(s)\right)=26 y-1+363 y^{3}-167 y^{2} \in \mathbb{R}[y]
$$

is a polynomial defining three horizontal asymptotes, $\widetilde{\mathcal{C}}_{i}, i=3,4,5$, of $\mathcal{C}$. One of these asymptotes is real $\left(\widetilde{\mathcal{C}_{3}}\right)$ and the other two $\left(\widetilde{\mathcal{C}}_{4}\right.$ and $\left.\widetilde{\mathcal{C}}_{5}\right)$ are complex (see Figures 4 and 6).

Step 3: $s_{1}=1$ is a root of $p_{22}(s)$ and $p_{12}\left(s_{1}\right) \neq 0$. Thus, additionally to the above asymptotes, we also get the vertical asymptote $\widetilde{\mathcal{D}}$ defined by the proper parametrization $\widetilde{\mathcal{M}}(t)=\left(p_{1}(0), t\right)=(1 / 8, t)$. We observe that this asymptote is defined implicitly by the polynomial $x-1 / 8$, and it corresponds to an infinity branch associated to the infinity point $(0: 1: 0)$ (see Figures 4 and 6). 

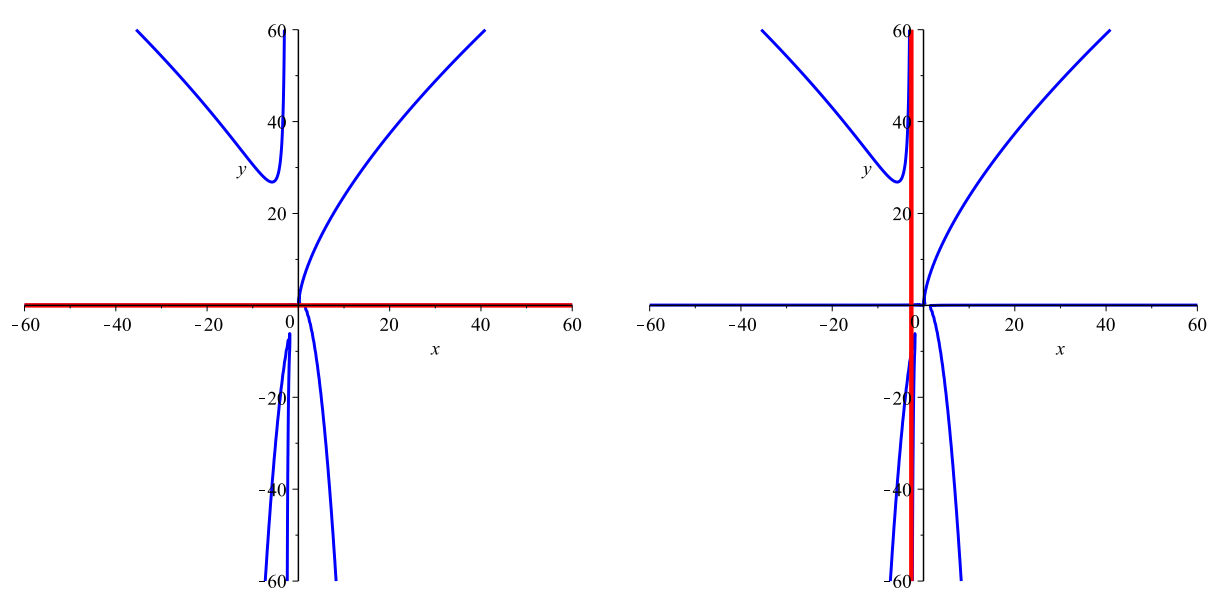

Figure 6: Curve $\mathcal{C}$ and asymptote $\widetilde{\mathcal{C}}_{3}$ (left), curve and asymptote $\widetilde{\mathcal{D}}$ (right).

Step 4: The algorithm returns the asymptotes $\widetilde{\mathcal{C}}_{i}, i=1, \ldots, 5$ and $\widetilde{\mathcal{D}}$.

Under the conditions of Theorem 3 , if $\operatorname{gcd}\left(m_{1}, p_{22}\right) \neq 1$, one can not apply statement 1 in Theorem 4 (similarly for statement 2). In this case, one applies the formulae presented in Theorem 3 , but modulo $m_{1}(s)$. That is, we use the polynomial $m_{1}(s)$ to carry out the arithmetic by computing polynomial remainders. Let us illustrate this idea with an example.

Example 7. Let $\mathcal{C}$ be the plane curve defined by the parametrization

$$
\mathcal{P}(s)=\left(\frac{s^{2}-1}{\left(s^{3}-2\right)^{2}}, \frac{2 s^{2}-s+1}{\left(s^{3}-2\right)}\right) \in \mathbb{R}(s)^{2} .
$$

Let $m(s)=s^{3}-2 \in \mathbb{R}[s]$. Observe that $m(s)$ divides $p_{12}(s)$ and $\operatorname{gcd}\left(m, p_{22}\right) \neq 1$. Thus, we can not apply Theorem 4. Let us apply the formulae presented in Theorem 3, but modulo $m(s)$. More precisely, for $\tau=a$, where $m(a)=0$, we compute

$$
\begin{aligned}
& a_{1}=\lim _{t \rightarrow a} \frac{p_{2}(t)}{p_{1}(t)^{1 / 2}}=\left(2 a^{2}-a+1\right) / \sqrt{a^{2}-1}=\left(2 a^{2}-a+1\right) / \alpha, \\
& a_{0}=\lim _{t \rightarrow a} p_{1}(t)^{1 / 2} f_{1}(t)=(1 / 3)\left(2 a^{3}-5 a+1\right) /\left((a-1)(a+1) a^{2}\right)
\end{aligned}
$$

where $f_{1}(t):=\frac{p_{2}(t)}{p_{1}(t)^{1 / 2}}-a_{1}$ and $\alpha:=\sqrt{a^{2}-1}$.

We obtain the asymptotes $\widetilde{\mathcal{C}}_{a}$ defined by the proper parametrization $\widetilde{\mathcal{Q}}_{a}(t)=\left(t^{2},\left(2 a^{2}-a+1\right) t / \alpha+(1 / 3)\left(2 a^{3}-5 a+1\right) /\left((a-1)(a+1) a^{2}\right)\right), \quad$ where $m(a)=0$. 
Observe that these asymptotes correspond to infinity branches associated to the infinity point (1:0:0) (see Remark 10).

By applying Remark 6 , we have that each asymptote $\widetilde{\mathcal{C}}_{a}$ is defined by the polynomial

$$
\begin{gathered}
\widetilde{f}_{a}(x, y)=\left(36 a^{10}-36 a^{9}+9 a^{8}+18 a^{7}-36 a^{6}+18 a^{5}-9 a^{4}\right) x+\left(-9 a^{8}-9 a^{4}+18 a^{6}\right) y^{2} \\
+\left(-42 a^{5}+6 a^{4}-6 a^{2}+12 a^{7}+30 a^{3}\right) y-1+20 a^{4}-4 a^{6}+10 a-4 a^{3}-25 a^{2}
\end{gathered}
$$

where $m(a)=0$. Using the properties of the resultants (see e.g. [8], we deduce that the polynomial

$$
\begin{gathered}
\tilde{f}(x, y)=\operatorname{resultant}_{a}\left(\widetilde{f}_{a}(x, y), m(a)\right)=-15625+877500 x-112500 y+2349000 y x-337500 y^{2} \\
+4374000 y^{2} x+4898880 y^{3} x+2624400 y^{4} x-567000 y^{3}-583200 y^{4}-349920 y^{5}-104976 y^{6} \\
-20470320 y^{2} x^{2}-7348320 y x^{2}+10400400 x^{2}+64700208 x^{3}
\end{gathered}
$$

defines implicitly the three asymptotes $\widetilde{\mathcal{C}}_{a}$. Note that just one of these asymptotes is real; in Figure 7 we have plotted the curve $\mathcal{C}$ and the real asymptote.
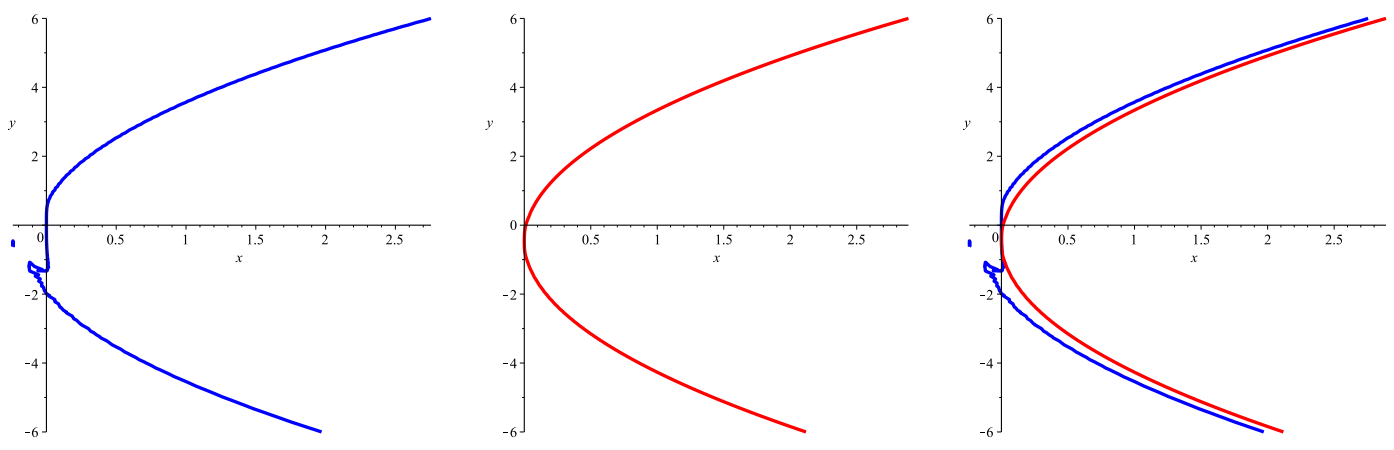

Figure 7: Curve $\mathcal{C}$ (left), asymptote (center), and both together (right)

\subsection{Experimental timings}

We finish this section by comparing the performance of algorithm Improvement Asymptotes Construction-Parametric Case (Method 1), algorithm Asymptotes ConstructionParametric Case, presented in [3] (Method 2) and algorithm Asymptotes Construction, presented in [2], which requires previous implicitation of the curve (Method 3).

We have implemented the algorithms, using Maplesoft 2016, on a Lenovo ThinkPad Intel(R) Core(TM) i7-7500U CPU @ 2.90 GHz and 16 GB of RAM, OS-Windows 10 
Pro. Then we have run them on a set of ten arbitrary parametric curves with different degrees and different numbers of infinity branches (their parametric expressions have been listed in the Appendix at Section 6; also, the implemented algorithms can be found in [4]). For each of these curves, we have recorded the degree, the number of infinity branches, and the running time (given in seconds of CPU) spent by each of the three methods. All these data are shown in the following table:

\begin{tabular}{|c||c|c|c|c|c|}
\hline Curve & Degree & $\begin{array}{c}\text { Infinity } \\
\text { branches }\end{array}$ & Method 1 & Method 2 & Method 3 \\
\hline \hline $\mathcal{C}_{1}$ & 4 & 4 & 0.032 & 0.031 & 0.015 \\
\hline $\mathcal{C}_{2}$ & 7 & 3 & 0.109 & 1.032 & 0.032 \\
\hline $\mathcal{C}_{3}$ & 7 & 5 & 0.203 & 0.234 & 0.032 \\
\hline $\mathcal{C}_{4}$ & 8 & 4 & 0.046 & 2.531 & 0.031 \\
\hline $\mathcal{C}_{5}$ & 12 & 6 & 0.019 & 9.968 & 0.093 \\
\hline $\mathcal{C}_{6}$ & 15 & 4 & 0.156 & $>200$ & 1.031 \\
\hline $\mathcal{C}_{7}$ & 17 & 5 & 0.688 & $>200$ & 0.859 \\
\hline $\mathcal{C}_{8}$ & 25 & 8 & 0.079 & 50.782 & 2.391 \\
\hline $\mathcal{C}_{9}$ & 35 & 9 & 0.015 & $>200$ & 82.641 \\
\hline $\mathcal{C}_{10}$ & 37 & 5 & 0.375 & $>200$ & 174.719 \\
\hline
\end{tabular}

In order to compare the three methods, we have marked in red the shortest running time for each curve. We observe that the new method (method 1) spends slightly longer times than the implicitation one (method 3) when we deal with low degree curves like $\mathcal{C}_{1}, \mathcal{C}_{2}$ and $\mathcal{C}_{3}$. However, its real power becomes clear when dealing with high degree curves, like $\mathcal{C}_{9}$ and $\mathcal{C}_{10}$. There, we get a significant improvement, since the existing algorithms spend much longer times than the new one.

Note also that these running times are related to the degree of the curve and the number of infinity branches. It makes sense, since the number of coefficients we need to compute for getting the asymptotes depends on both parameters.

\section{Conclusion}

The main result of this paper, Theorem 3, provides a way to determine the generalized asymptotes of a rational curve by only computing some simple limits of functions constructed from the given parametrization. We prove this theorem and develop an efficient algorithm which determines all the g-asymptotes avoiding the laborious computation of Puiseux series and infinity branches. The comparison with other existing methods shows that this one reduces significantly the computation time, specially when we deal with high degree curves. As a complement, some corollaries are derived that allow us to obtain the horizontal and vertical asymptotes in an extremely simple way. 
The method proposed requires the computation of roots of certain polynomials. In the case that these roots can not be computed in an exact way, we overcome the problem by using families of conjugate points and polynomial remainders. These techniques are proved to work on several illustrative examples.

It is important to stress that this procedure can be trivially applied for dealing with rational parametrizations of curves in $n$-dimensional space (see Example 5). Thus, the present paper yields a remarkable improvement of the methodology developed in [3] (see Section 5).

As a future work, we aim to extend the notion of g-asymptote to the study of the asymptotic behavior of algebraic surfaces. We look for surfaces which approach a given

one of higher degree, when "moving to infinity", that is, when some of the coordinates take infinitely large values. The ideas introduced in this paper might provide the foundations for efficient methods that allow us to compute those "asymptotic surfaces".

\section{Appendix}

In Subsection 4.3, we compare the running times of three different methods for computing asymptotes. For this purpose, we run them on a set of ten arbitrary parametric curves with different degrees and different numbers of infinity branches. The parametric expressions of those ten curves are the following:

- $\mathcal{P}_{1}(t)=\left(\frac{2 t^{3}-t-1}{t^{4}-t^{2}+1}, \frac{2 t^{3}+t^{2}+1}{t^{4}-t^{2}+1}\right)$

- $\mathcal{P}_{2}(t)=\left(\frac{t^{3}+2 t-1}{t^{7}-6 t^{6}+14 t^{5}-20 t^{4}+25 t^{3}-22 t^{2}+12 t-8}, \frac{2 t^{3}+t^{2}+1}{t^{4}+5 t^{2}-4 t^{3}-4 t+4}\right)$

- $\mathcal{P}_{3}(t)=\left(\frac{t^{2}+1}{t^{7}-3 t^{5}+3 t^{3}-t+t^{4}-2 t^{2}+1}, \frac{2 t^{3}+5 t^{2}+1}{t^{5}-2 t^{3}+t+t^{2}-1}\right)$

- $\mathcal{P}_{4}(t)=\left(\frac{t-1}{t^{8}-4 t^{4}+4}, \frac{t^{3}}{t^{4}-2}\right)$.

- $\mathcal{P}_{5}(t)=\left(\frac{t-1}{t^{12}+4 t^{6}+4}, \frac{t^{3}-t+1}{t^{6}+2}\right)$

- $\mathcal{P}_{6}(t)=\left(\frac{t^{5}+t+2 t^{2}-1}{p_{12}(t)}, \frac{2 t^{4}+t^{2}+1-t^{3}}{p_{22}(t)}\right)$

$p_{12}(t)=t^{15}+8 t^{13}+25 t^{11}+40 t^{9}+35 t^{7}+16 t^{5}-3 t^{14}-16 t^{12}-35 t^{10}-40 t^{8}-$ $25 t^{6}-8 t^{4}+3 t^{3}-t^{2}$

$p_{22}(t)=t^{8}-2 t^{7}+4 t^{6}-6 t^{5}+6 t^{4}-6 t^{3}+4 t^{2}-2 t+1$. 


$$
\begin{aligned}
& \text { - } \mathcal{P}_{7}(t)=\left(\frac{t^{8}+2 t^{4}-t^{2}-t-1}{t^{17}+10 t^{15}+42 t^{13}+96 t^{11}+129 t^{9}+102 t^{7}+44 t^{5}+8 t^{3}}, \frac{t^{2}+1+t^{7}+t^{6}-t^{5}}{t^{7}+6 t^{5}+12 t^{3}+8 t}\right) \\
& \text { - } \mathcal{P}_{8}(t)=\left(\frac{t^{2}-t+5}{t^{16}+4 t^{9}+4 t^{2}}, \frac{t^{10}-t^{5}+1+t}{t^{7}+2 t}\right) \\
& \text { - } \mathcal{P}_{9}(t)=\left(\frac{2 t^{10}+t^{8}-t^{7}-t^{2}-t-1}{p_{12}(t)}, \frac{t^{2}+1+t^{6}}{p_{22}(t)}\right) \\
& p_{12}(t)=16 t-32+96 t^{5}-192 t^{4}+248 t^{9}-496 t^{8}+360 t^{13}-720 t^{12}+321 t^{17}- \\
& 642 t^{16}+t^{33}-2 t^{32}+12 t^{29}-24 t^{28}+62 t^{25}-124 t^{24}+180 t^{21}-360 t^{20} \\
& p_{22}(t)=t^{6}+t^{4}+2 t^{2}+2 . \\
& \text { - } \mathcal{P}_{10}(t)=\left(\frac{t^{8}-t-1}{p_{12}(t)}, \frac{2 t^{4}+t^{2}+1+t^{7}+t^{1} 0-t^{5}}{p_{22}(t)}\right) \\
& p_{12}(t)=256 t^{3}+3328 t^{5}+20224 t^{7}+200032 t^{11}+76288 t^{9}+386848 t^{13}+571312 t^{15}+ \\
& 658048 t^{17}+598417 t^{19}+25 t^{35}+2116 t^{31}+39550 t^{27}+247684 t^{23}+432073 t^{21}+ \\
& t^{37}+292 t^{33}+10654 t^{29}+112084 t^{25} \\
& p_{22}(t)=t^{15}+7 t^{13}+21 t^{11}+35 t^{9}+35 t^{7}+21 t^{5}+7 t^{3}+t .
\end{aligned}
$$

The computation of the asymptotes with the three methods has been carried out using Maplesoft 2016 and can be found in [4].

\section{Acknowledgements}

The author S. Pérez-Díaz is partially supported by FEDER/Ministerio de Ciencia, Innovación y Universidades - Agencia Estatal de Investigación/MTM2017-88796-P (Symbolic Computation: new challenges in Algebra and Geometry together with its applications).

The author S.Pérez-Díaz belongs to the Research Group ASYNACS (Ref. CCEE2011/R34).

\section{References}

[1] Blasco A., Pérez-Díaz S., Asymptotes and Perfect Curves. Computer Aided Geometric Design. Vol. 31, Issue 2, pp. 81-96 (2014).

[2] Blasco A., Pérez-Díaz S., Asymptotic Behavior of an Implicit Algebraic Plane Curve. Computer Aided Geometric Design. Vol. 31, Issue 7-8. pp. 345-357 (2014).

[3] Blasco A., Pérez-Díaz S., Asymptotes of Space Curves. Journal of Computational and Applied Mathematics. Vol. 278. pp. 231-247 (2015). 
[4] Blasco A., Pérez-Díaz S., Recent Advances in the Computation of Asymptotes for Parametric Curves [Data set]. Journal of Computational and Applied Mathematics. Zenodo. http://doi.org/10.5281/zenodo.3257703 (2019).

[5] Kečkić J.D., A Method for Obtaining Asymptotes of Some Curves. The Teaching of Mathematics. Vol. III, 1. pp. 53-59 (2000).

[6] Maxwell E. A., An Analytical Calculus. Vol. 3. Cambridge (1962).

[7] Pérez-Díaz S., Computation of the Singularities of Parametric Plane Curves. Journal of Symbolic Computation. Vol. 42/8. pp. 835-857 (2007).

[8] Sendra J.R., Winkler F., Pérez-Díaz S., Rational Algebraic Curves: A Computer Algebra Approach. Series: Algorithms and Computation in Mathematics. Vol. 22. Springer Verlag (2007).

[9] Zeng G., Computing the Asymptotes for a Real Plane Algebraic Curve. Journal of Algebra. Vol. 316. pp. 680-705 (2007). 